\title{
Bridge principle for constant and positive Gauss curvature surfaces.
}

\author{
LAURENT HAUSWIRTH
}

\section{Introduction.}

Parmi toutes les surfaces bordées par une même courbe de Jordan fermée, quelles sont celles qui minimisent l'aire? Que dire de leur régularité?

Ce problème porte le nom du physicien belge Joseph Antoine Ferdinand Plateau. En 1850, il observe expérimentalement des solutions en immergeant des structures métalliques à l'image de ces courbes dans de l'eau savonneuse.

Ces deux questions vont tenir en éveil les mathématiciens durant plus d'un siècle. Riemann, Schwarz, Weierstrass, Lamarle et beaucoup d'autres au $19^{\text {eme }}$ siècle obtiennent des résultats dans de nombreux cas particuliers.

1930 et 1931, l'approche moderne de Jesse Douglas et Tibor Radó par le calcul des variations permet une première réponse globale.

Ils montrent l'existence d'un minimum pour l'aire dans l'ensemble des surfaces homéomorphes au disque et bordées par une même courbe rectifiable $\gamma$ de $\mathbb{R}^{n}$.

L'équation d'Euler associée à la variation de l'aire montre que toutes les solutions de ce problème sont des surfaces minimales, c'est à dire des surfaces de courbure moyenne nulle.

Les solutions de Douglas-Radó sont des surfaces minimales, mais dans de nombreux cas, pour certaines courbes, elles ne sont pas un minimum absolu pour l'aire.

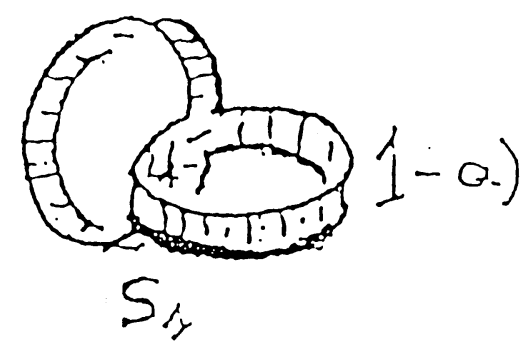

Fig 1

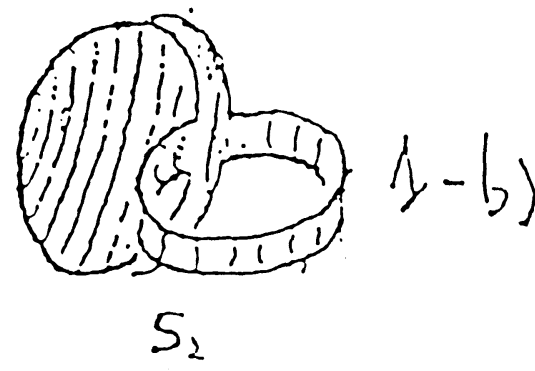


Il est facile par exemple de construire un contour comme dans la figure 1, où la surface de genre 1 (Voir Figure 1-a)) est d'aire plus petite que le disque (Voir Figure 1-b)) solution de Douglas-Radó.

Cet exemple suggère à J.Douglas une reformulation du problème de Plateau. On cherche dorénavant à construire une surface minimale bordée par une courbe de Jordan donnée en prescrivant la topologie de la solution.

Il faudra attendre les années 60 avec les travaux de Federer, Fleming et Almgren sur les courants pour bien comprendre l'existence et la régularité des surfaces d'aire minimisante.

Plus généralement, on s'intéresse à l'ensemble des surfaces minimales bordées par une même courbe. Quelle est leur nombre, leur topologie? Quelles sont celles qui minimisent l'aire?...

Dans le cadre de cette recherche, R.Courant dans son livre [5] en 1950, propose un principe du pont dont il suggère une preuve. Les deux exemples qui suivent illustrent bien l'intérêt de cette construction. Le principe du pont, c'est la possibilité de connecter par un ruban $\mathrm{P}$ d'épaisseur $\varepsilon>0$, deux surfaces minimales $S_{1}$ et $S_{2}$, bordées par $\gamma_{1}$ et $\gamma_{2}$. Par petites deformations de $S_{1} \bigcup S_{2} \bigcup P$ on obtient une nouvelle surface minimale $S^{\varepsilon}$, bordée par une courbe $\gamma_{\varepsilon}$ constituée de l'union de $\gamma_{1}, \gamma_{2}$ et de deux courbes parallèles éloignées d'au plus une distance $\varepsilon>0$ (Voir Figure 2). De plus, on demande à $S^{\varepsilon}$ de converger vers $S_{1} \bigcup S_{2}$ quand $\varepsilon \rightarrow 0$.

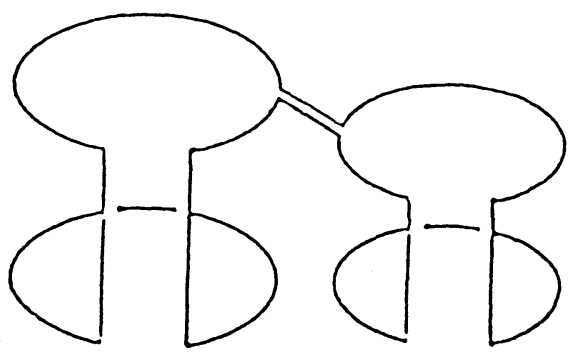

Fig 2

Certains contours de $\mathbb{R}^{3}$ possèdent au moins deux solutions de DouglasRadó. Par exemple, pour deux cercles dans des plans parallèles, on peut ajuster la distance entre les plans pour que la caténoide moins un petit ruban soit d'aire égale à la surface des deux disques plus le ruban (Voir Figure 3). Ces deux surfaces ont la topologie du disque et sont toutes deux 
minimales.
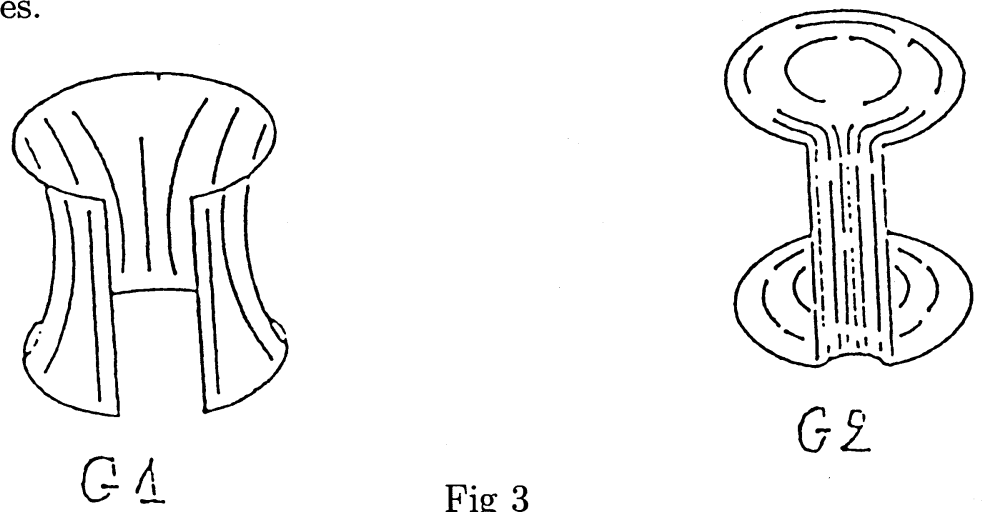

Fig 3

On va construire, grâce à ce principe, une courbe rectifiable qui va border un nombre infini de surfaces minimales. Elles auront toutes la topologie du disque.

On considère le contour $\gamma_{1}$ de la figure 3 et un contour $\gamma_{2}$ géométriquement similaire mais de taille différente. Chacune de ces deux courbes est le bord d'au moins deux surfaces minimales dont la topologie est celle du disque. Par le principe du pont, on obtient un contour (Voir Figure 2)) qui borde au moins quatre surfaces minimales, qui de part et d'autre du pont ont soit l'apparence de $G_{1}$, soit celle de $G_{2}$. On réitère cette opération $N_{0}$ fois. La courbe obtenue borde $2^{N_{0}}$ surfaces minimales. Par décroissance géométrique de la taille des contours et des ponts, on obtient à la limite une courbe rectifiable qui borde un nombre infini de surfaces minimales, toutes topologiquement des disques (Voir Figure 4).

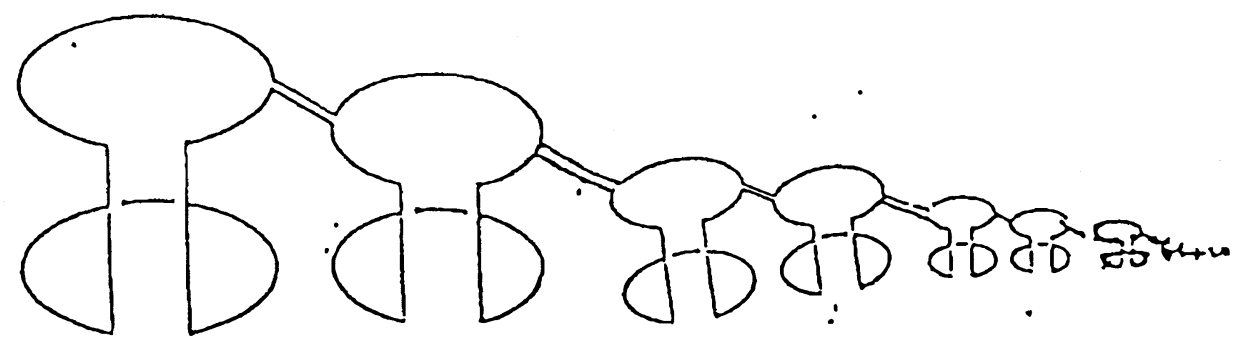

Fig 4

On doit à W.Fleming [7] l'exemple suivant sur la topologie d'une solution du problème de Plateau. A partir de la courbe de la figure 1, et d'une application itérée du principe du pont comme ci-dessus, on obtient la courbe $\gamma_{3}$ de la figure 5 -a). 
Cette courbe borde un disque minimisant en ne connectant que des surfaces de genre 0, de type $S_{2}$ comme dans la figure 5-b). En remplacant la première surface, par une surface de type $S_{1}$, on obtient une surface minimale dont la topologie est celle du disque à qui on a rajouté une anse (Voir Figure 5-c)). Cette dernière surface est d'aire plus petite que la première. Dans la figure 5-d), on obtient une autre surface d'aire inférieure et de genre 2. En réitérant cette opération, on a une suite minimisante de surfaces minimales pour l'aire et dont le genre augmente. Pour cette courbe rectifiable la solution du problème de Plateau est de genre infini.

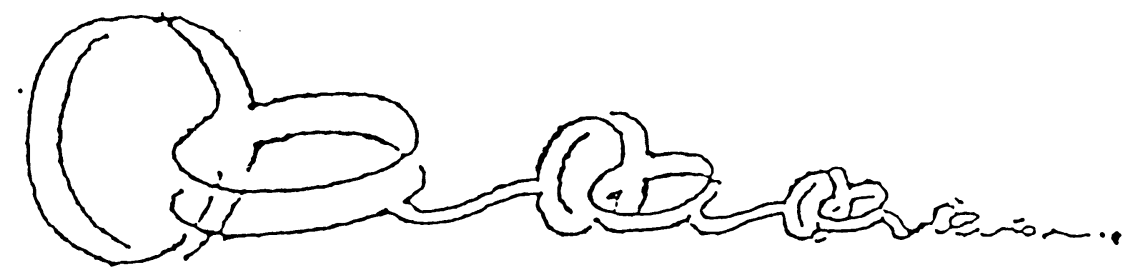

Fig 5-a

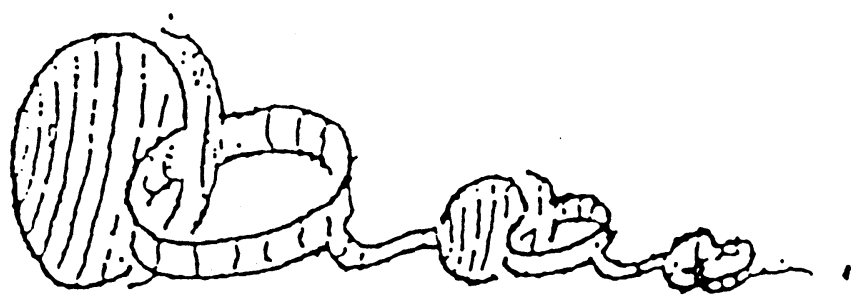

Fig 5-b

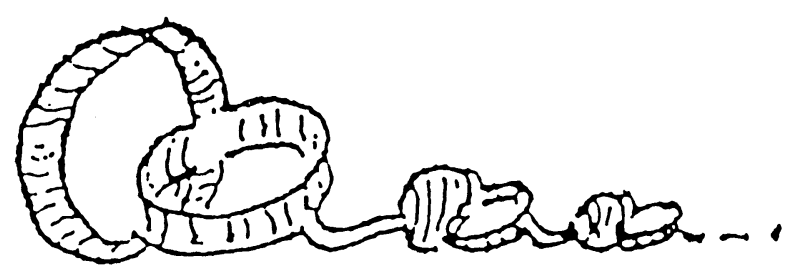

Fig 5-c 


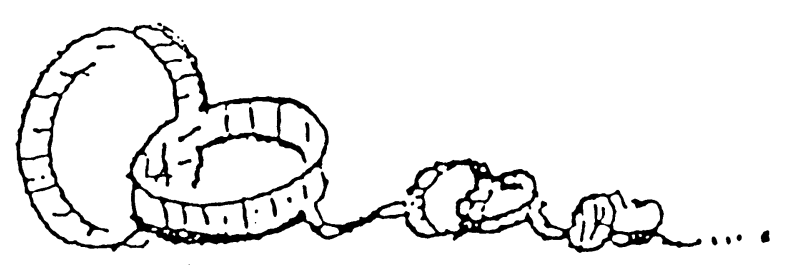

Fig 5-d [13]

Dans l'esprit de ces deux constructions, P.Lévy [14] en 1948, construit des exemples et offre des arguments pour l'existence du principe du pont. Il faudra attendre 1982, pour que W.Meeks et S-T Yau [16] publient une démonstration d'un principe du pont pour les surfaces stables et orientables de $\mathbb{R}^{3}$ qui justifie la construction de P.Lévy. En 1984, Lawson et Michelson [15] publient un principe du pont faible pour les surfaces orientables et stables de $\mathbb{R}^{n}$ qui ne permet pas de justifier les exemples de non unicité évoqués. En effet, leur construction nécessite que le ruban ou le pont arrive tangentiellement aux surfaces considérées. $\mathrm{Si} G_{1}$ et $G_{2}$ sont bordées par la même courbe, le pont ne pourra pas être tangent aux deux à la fois. Jusqu'à présent, aucune de ces démonstrations ne convient pour un principe du pont dans le cas de surfaces instables. En 1987, N.Smale [20] publie un principe du pont faible pour toutes surfaces minimales régulières dans n'importe quelles dimensions et codimensions. Sa preuve reste valable dans le cas des surfaces instables de nullité 0 et pour les surfaces de courbure moyenne constante non nulle. Sa démonstration nécessite également un ruban tangent aux surfaces.

C'est B.White [23] et [?] qui établit le théorème du pont fort pour les surfaces minimales stables et instables qui justifie les constructions de P.Lévy, mais aussi de J.Hass [9] et P.Hall [10]. Il construit un pont en contraignant le bord du ruban à rester dans une sous- variété que l'on peut choisir transverse aux surfaces qu'il connecte.

Une question non résolue aujourd'hui concerne l'existence d'une surface minimale de genre 1 bordée par deux courbes convexes contenues dans deux plans parallèles (Voir Figure 6-a)). W.Meeks conjecture qu'une telle surface n'existe pas. Grâce au principe du pont fort, on peut montrer que de telles surfaces peuvent exister si les bords ne sont pas convexes. En prenant deux fois le même morceau de caténö̈de stable, bordée par deux cercles dans des plans parallèles, on peut construire un pont en haut et un autre en bas (Voir Figure 6-b)). La surface minimale qui en résulte est bien de genre 1, mais 
elle est bordée en haut et en bas par des courbes non convexes en forme d'haltères (Voir Figure 6-c)).

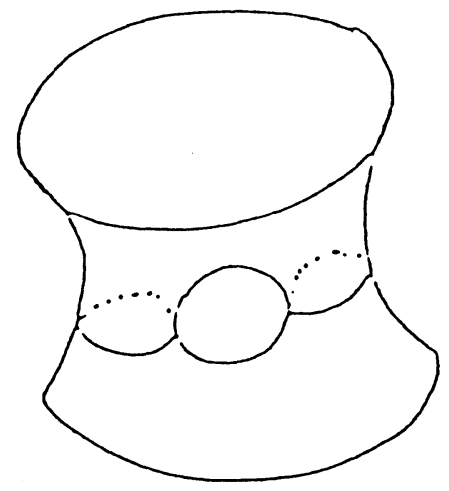

Fig 6-a
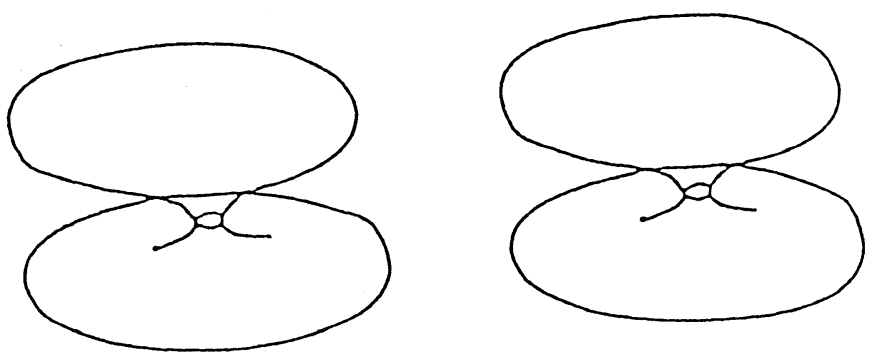

Fig 6-b

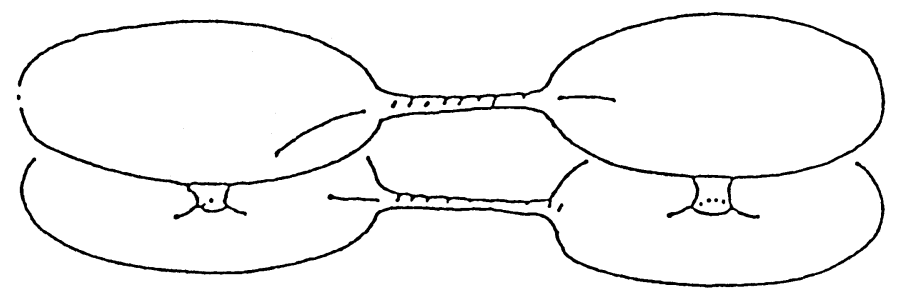

Fig 6-c

Tous ces theorèmes concernent les surfaces de courbure moyenne constante. Pour les surfaces de $\mathbb{R}^{3}$, il existe une autre fonction de courbure: la courbure Gaussienne ou courbure de Gauss. Alors que l'équation liée au problème de trouver une surface de courbure moyenne $\mathrm{H}$ prescrite est de type quasi-linéaire, la recherche d'une surface à courbure de Gauss $K>0$ prescrite conduit à une équation fortement non linéaire, de type MongeAmpère. 
Le problème de Dirichlet pour les graphes de courbure de Gauss constante et positive est résolu sur des domaines convexes par Cafarelli, Nirenberg et Spruck [4] en 1984. Ils utilisent la méthode de continuité dont le point central est une estimée $C^{2}$ difficile à obtenir. En 1992, Hoffman, Rosenberg et Spruck [11] étendent ce résultat pour les graphes au-dessus des anneaux plans.

B.Guan et J.Spruck [2] montrent par la même méthode que deux courbes convexes dans des plans parallèles bordent une K-surface dont la topologie est celle de l'anneau. Ce résultat n'est toujours pas montré dans le cas des $\mathrm{H}$-surfaces.

Cependant, sauf pour quelques cas particuliers, on ne connait pas le nombre de K-surfaces que borde une courbe. H.Brezis, J.M Coron [3] et M.Struwe [21] ont montré que pour une courbe donnée l'existence d'une "petite" H-surface, impliquait l'existence d'une "grande" H-surface.

$\mathrm{H}$. Rosenberg conjecture que de nombreux théorèmes valables dans le cadre des $\mathrm{H}$-surfaces, restent vrai dans le cadre des $\mathrm{K}$-surfaces (et vice-versa). Dans l'esprit de ce principe, cette thèse montre qu'il existe un principe du pont faible pour les K-surfaces. Le ruban $\mathrm{P}$ devra être tangent aux surfaces. On ne peut pas contraindre le bord du pont à rester dans une sous-variété transverse aux surfaces. Par exemple, si une telle construction était possible, on connecterait deux calottes sphériques bordées par deux cercles situés dans un même plan (Voir Figure 7). On obtiendrait alors une K-surface bordée par une courbe plane possédant des points d'inflexions, obstruction à l'existence de surface de courbure de Gauss positive.

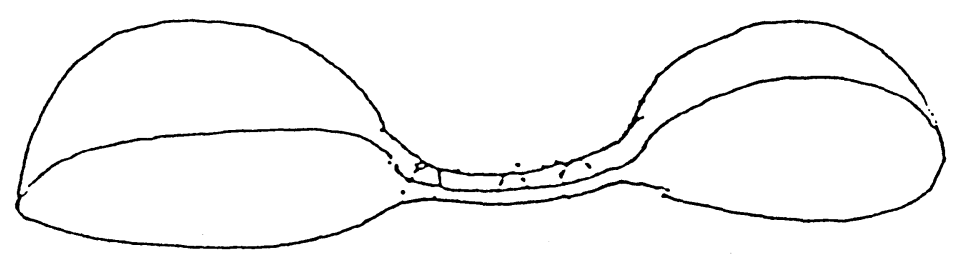

Fig 7

La preuve de ce théorème est similaire à celle de N.Smale [20], dans le cas des $\mathrm{H}$-surfaces. On reformule le problème en un théorème de point fixe de Schauder en linéarisant l'équation. Ce point de vue a été introduit par R.Schoen et utilisé avec succès par Kapouleas $[\mathrm{K}]$ pour construire de nouvelles surfaces de courbure moyenne constante non nulle. Par les mêmes méthodes, M.Traizet [22] a construit des surfaces minimales complètes et plongées en recollant des surfaces de Scherk simplement périodiques. Il était 
donc pertinent de s'intéresser à cette méthode dans le cadre des K-surfaces. Remerciements. Je remercie Harold Rosenberg pour son soutien et ses nombreux conseils tout au long de ce travail.

\section{Theorem.}

In 1987 N. Smale proved [20] a bridge principle for constant mean curvature hypersurfaces of $\mathbb{R}^{n}$ without Jacobi fields. In this paper we prove, the same principle for surfaces immersed in $\mathbb{R}^{3}$ with constant and positive Gauss curvature without Jacobi fields. More precisely, we prove the following result:

Theorem 1.1. Let $S_{1}, S_{2}$ be compact, immersed hypersurfaces of $\mathbb{R}^{3}$, with boundary, of constant Gauss curvature $K\left(S_{i}\right)_{i=1,2}=C>0$, in particular they are strictly locally convex. Assume that $\left(S_{i}\right)_{i=1,2}$ are analytic up to their boundaries (with $0<\beta<1$ ) and these surfaces do not have normal Jacobi fields which vanish on the boundary.

Let $q_{1}$ and $q_{2}$ be points of $\partial S_{1}$ and $\partial S_{2}$.

Then we can find an embedded path $\gamma$, connecting the boundaries of $S_{1}, S_{2}$, intersecting $S_{1} \cup S_{2}$ only at its end points such that for any tubular neighborhood $T$ of $\gamma$, there exists a bridge inside $T$, connecting $\partial S_{1}$ and $\partial S_{2}$ such that the resulting configuration spans $M$, a constant Gauss curvature surface with $K(M)=C>0$, which is diffeomorphic to $S_{1}$ and $S_{2}$ joined by a thin strip.

Moreover $M$ is a small perturbation of $S_{1}$ and $S_{2}$ joined by the strip.

Without loss of generality, up a dilation, we may assume that the Gauss curvature $K\left(S_{i}\right)_{i=1,2}=1$. By a bridge, we mean two nearby curves and by a strip, a diffeomorphic copy of an interval $\times$ interval.

To prove this theorem, we apply the same technique as N.Smale [20] for $\mathrm{H}$-surfaces. We construct a family of approximate solutions $M^{\varepsilon}$ of class $C^{4, \beta}$ with a certain path $\gamma$, and a tubular neighborhood $\mathrm{T}$ of $\gamma$. The parameter $\varepsilon>0$ is the width of the strip $S^{\varepsilon}$. We attach this strip to $\left(S_{i}\right)_{i=1,2}$ in a neighborhood of $\left(q_{i}\right)_{i=1,2}$. We need the surfaces to be analytic up to their boundaries to make an analytic extension in a neighborhood of $q_{1}, q_{2}$ (see section 2). This will be the only place where we need analytic surfaces.

We look for a solution as a normal perturbation of $M^{\varepsilon}$ by linearizing the Gauss curvature and reformulating the equations as a fixed point problem. Since the linearized operator is strictly elliptic when $M^{\varepsilon}$ is strictly locally 
convex and the quadratic term is slighty different, we contruct $M^{\varepsilon}$ very carefully.

We construct $M^{\varepsilon}$ so that $|K-1|_{C^{0}\left(M^{\varepsilon}\right)}=|K-1|_{C^{0}\left(S^{\varepsilon}\right)} \leq c \varepsilon^{2}$, in particular $M^{\varepsilon}$ is convex (see section 3 ) and $\mathrm{E}(\mathrm{u})$ is small enough (see section 2).

We choose a path $\gamma$, which is alternatively plane and on spheres with radius 1 (see section 3 ).

For each $\gamma_{1}$, an embedded path as in theorem 1.1 and each tubular neighborhood $T_{1}$ of $\gamma_{1}$, we can construct a strip in $T_{1}$, along an embedded path $\gamma_{0} \subset T_{1}$, which is composed of planar and spherical arcs by jumping from one sphere to an other by planar curves.

\section{Notations.}

For $\Psi: S \longrightarrow \mathbb{R}^{3}$ a compact $C^{4, \beta}$ hypersurface immersed in $\mathbb{R}^{3}$ with boundary $C^{4, \beta}$, TS and NS denote the tangent and normal bundles of $\mathrm{S}$, $T_{q} S$ and $N_{q} S$ the corresponding fibers at $q \in S$.

$\mathrm{N}$ will be the unit normal vector field in $\mathbb{R}^{3}$ of $\mathrm{S}$, which orients the surface, $N \in C^{3, \beta}\left(S, \mathbb{R}^{3}\right)$ and if $U \in C^{3, \beta}(N S)$ is a section, there exists $u \in C^{3, \beta}(S, \mathbb{R})$ such that $U=u N$.

A is the symmetric operator associated to the second fundamental form. Then there exists at each point q of $\mathrm{S}$, an orthonormal basis $\left(e_{i}(q)\right)_{i=1,2}$ of $T_{q} S$, eigenvectors associated to $\left(\kappa_{i}\right)_{i=1,2}$, the eigenvalues of the operator. A surface strictly locally convex satisfies $\left(\kappa_{i}\right)_{i=1,2} \neq 0$ and $\kappa_{1}, \kappa_{2}$ are of the same sign.

We orient the surface such that $\left(\kappa_{i}\right)_{i=1,2}>0$.

$\mathrm{K}(\mathrm{q})$ is the Gauss curvature at $\mathrm{q}$ of $\mathrm{S}$ and $K \in C^{2, \beta}(S, \mathbb{R})$ with

$$
K=\operatorname{det} A=\kappa_{1} \kappa_{2}
$$

Let $u \in C_{0}^{3, \alpha}(S)$ be a $C^{3, \alpha}$ real function on S, zero on the boundary $\partial S$ with $0<\alpha \leq \beta$. If $|u|_{C^{1}}$ is sufficiently small then $\mathrm{u}$ induces a $C^{3, \alpha}$ immersion $\Psi_{u}=\Psi+u N: S \longrightarrow \mathbb{R}^{3}$ and $\Psi_{u} \equiv \Psi$ on $\partial S$. In the following we often identify $\mathrm{S}$ with $\Psi(S)$ and we note $S_{u}=\Psi_{u}(S)$ the small normal perturbation of S.

Let $\varepsilon_{0}>0$ be small enough so that for $u \in D=\left\{u \in C_{0}^{3, \alpha}(S):|u|_{C^{1}} \leq\right.$ $\left.\varepsilon_{0}\right\}, S_{u}$ is a surface immersed.

$\mathrm{K}(\mathrm{u})$ denote the Gauss curvature of $S_{u}, K(u) \in C^{1, \alpha}(S)$, moreover

$$
K: D \subset C_{0}^{3, \alpha}(S) \longrightarrow C^{1, \alpha}(S)
$$


is a $C^{\infty}$ map of these Banach spaces. The Jacobi operator associated to the Gauss curvature of $\mathrm{S}$ is (see [19])

$$
L u=\frac{d K}{d t}(t u)_{\mid t=0}=\operatorname{div} T_{1} \nabla u+2 K H u
$$

where $\mathrm{H}$ is mean curvature on $\mathrm{S}$ and $T_{1}=\operatorname{trace}(A) I-A$. L is a uniformly elliptic operator, self adjoint, Fredholm of index 0 when $\mathrm{S}$ is strictly locally convex. A Jacobi field is a normal variation $u N$ of $S, u=0$ on $\partial S$, which is a solution of the equation $L u=0$.

\section{Reformulation of the bridge theorem.}

We reformulate theorem 1.1 as an elliptic boundary value problem on the approximate solution $M^{\varepsilon}$. We construct $M^{\varepsilon}$ (see section 3) so that $\Psi(\varepsilon): M^{\varepsilon} \longrightarrow \mathbb{R}^{3}$, the immersion, satisfies $C_{0}^{-1} \leq|D \Psi(\varepsilon)| \leq C_{0}$ and $|\Psi(\varepsilon)|_{C^{4, \beta}} \leq C_{0}$ for a constant $C_{0}>0$ large enough, independent of $\varepsilon>0$ the width of the strip.

Let $\varepsilon_{0}>0$ be chosen (depending only on $C_{0}$ ) so that $|u|_{C^{1}} \leq \varepsilon_{0}$ implies that $\Psi(\varepsilon)+u N$ is an immersion and let $D=\left\{u \in C_{0}^{3, \alpha}\left(M^{\varepsilon}\right):|u|_{C^{1}} \leq \varepsilon_{0}\right\}$ with $0<\alpha \leq \beta$.

To prove theorem 1.1, we would like to find $u \in D \subset C_{0}^{3, \alpha}\left(M^{\varepsilon}\right)$ such that $K(u)=1$ and show that $u$ is small as $\varepsilon \rightarrow 0$.

Applying Taylor's theorem, we have for $u \in D$ :

$$
K(u)=K+L u+E(u)
$$

where

$$
\begin{aligned}
K & =K(0)=\text { Gauss curvature of } M^{\varepsilon}, \\
L u & =\left.\frac{d K}{d t}(t u)\right|_{t=0}=\operatorname{div} T_{1} \nabla u+2 K H u, \\
E(u) & =\int_{0}^{1}(1-\tau) \frac{d^{2} K}{d \tau^{2}}(\tau u) d \tau .
\end{aligned}
$$

$L$ is a uniformly elliptic operator since $M^{\varepsilon}$ is strictly locally convex for each $\varepsilon>0$ small with the coefficients of $T_{1}$ depending on the coefficients of the second fundamental form (see section 1). In particular if $\operatorname{support}(u) \subset S_{i}$, $\mathrm{i}=1,2$, then $L u=L^{S_{i}} u$, where $L^{S_{i}}$ is the Jacobi operator for $S_{i}$. As we are assuming $L^{S_{i}}$ has no kernel we will see that $\mathrm{L}$ has no kernel for $\varepsilon$ sufficiently small.

In section 6 we prove: 
Bridge principle for constant and positive Gauss curvature surfaces 507

Lemma 6.1. There exists positive constants $\varepsilon_{1}$ and $d_{0}$ such that for all $\varepsilon<\varepsilon_{1}$

$$
\operatorname{dist}(\operatorname{spec}(L), 0) \geq d_{0}>0
$$

$\varepsilon_{1}$ and $d_{0}$ depend only on $M a x_{M^{\varepsilon}}|2 K H|$ and $\min _{i=1,2} \operatorname{dist}\left(\operatorname{spec}\left(L^{S_{i}}\right), 0\right), \operatorname{spec}(L)=$ set of eigenvalues of $L=\{\lambda \in \mathbb{R} \mid(L+\lambda) u=0$ has a non zero solution $u \in$ $\left.C_{0}^{3, \alpha}\left(M^{\varepsilon}\right)\right\}$.

We also note that $L: C_{0}^{3, \alpha}\left(M^{\varepsilon}\right) \longrightarrow C^{1, \alpha}\left(M^{\varepsilon}\right)$ is a self adjoint uniformly elliptic (linear) operator. Then it is standard (see [18]), that $\operatorname{spec}(L)$ is a discrete set bounded from below and increasing to $\infty$.

Using lemma 6.1 we formulate the problem as a fixed point problem:

$$
\begin{aligned}
K(u)=1 & \Leftrightarrow K+L u+E(u)=1 \\
& \Leftrightarrow u=-L^{-1}(K-1)-L^{-1}(E(u)) \Leftrightarrow u=J(u)
\end{aligned}
$$

where $J(u)=-L^{-1}(K-1)-L^{-1}(E(u))$.

By standard elliptic theory

$$
L^{-1}: C^{1, \alpha}\left(M^{\varepsilon}\right) \longrightarrow C_{0}^{3, \alpha}\left(M^{\varepsilon}\right)
$$

is a continuous map, for any $0<\alpha \leq \beta, \varepsilon \leq \varepsilon_{1}$. $(K-1) \in C^{1, \alpha}\left(M^{\varepsilon}\right)$ and

$$
E: D \subset C_{0}^{3, \alpha}\left(M^{\varepsilon}\right) \longrightarrow C^{1, \alpha}\left(M^{\varepsilon}\right)
$$

is a continuous map for any $0<\alpha \leq \beta$. Therefore:

$$
J: D \subset C_{0}^{3, \alpha}\left(M^{\varepsilon}\right) \longrightarrow C_{0}^{3, \alpha}\left(M^{\varepsilon}\right)
$$

is a continuous map for any $0<\alpha \leq \beta, \varepsilon \leq \varepsilon_{1}$.

We recall the Schauder fixed point theorem (see [8]). If $\mathrm{P}$ is a convex, compact set of a banach space $\mathrm{B}$ and if $J: P \longrightarrow P$ is a continuous map, then $\mathrm{J}$ has a fixed point.

We solve $J(u)=u$ for some $u \in D$ by using the Schauder fixed point theorem.

The appropriate sets to look at are as follows. For $\varepsilon \leq \varepsilon_{1}, 0<\alpha \leq \beta, 0<$ $\sigma \leq \frac{1}{2}$, we define $P(\varepsilon, \sigma, \alpha)$ :

$$
\begin{aligned}
P(\varepsilon, \sigma, \alpha)=\left\{u \in C_{0}^{3, \alpha}\left(M^{\varepsilon}\right):|u|_{C^{0}} \leq \varepsilon^{2,75-\sigma},\right. & \\
& \left.|u|_{C^{3, \alpha}} \leq \varepsilon^{-0.25-\alpha-\sigma},|u|_{H^{2}} \leq \varepsilon^{1,75-\sigma}\right\} .
\end{aligned}
$$

Clearly $P(\varepsilon, \sigma, \alpha)$ is a convex, closed and bounded set in $C_{0}^{3, \alpha}\left(M^{\varepsilon}\right)$. Now for $\alpha^{\prime}<\alpha, P(\varepsilon, \sigma, \alpha) \subset C_{0}^{3, \alpha^{\prime}}\left(M^{\varepsilon}\right)$ and is in fact compact as the inclusion 
$C_{0}^{3, \alpha}\left(M^{\varepsilon}\right) \longrightarrow C_{0}^{3, \alpha^{\prime}}\left(M^{\varepsilon}\right)$ is compact (see [8]). It will follow from lemma 5.3 that if $u \in P(\varepsilon, \sigma, \alpha)$ then $|u|_{C^{1}} \leq c \varepsilon^{1,75-\alpha-\sigma}$ for some constant depending only on $C_{0}$. So $P(\varepsilon, \sigma, \alpha) \subset D$ for $\varepsilon<\varepsilon_{2}$ for $\varepsilon_{2}>0$ small enough (so $\mathrm{J}$ is defined on $P(\varepsilon, \sigma, \alpha))$.

We reformulate theorem 1.1 as follows:

Theorem 2.1. There exists $\bar{\alpha}>0, \bar{\sigma}>0$ and $\bar{\varepsilon}>0$ depending on $C_{0}, d_{0}, S_{1}, S_{2}$ such that for all $\varepsilon<\bar{\varepsilon}$ :

$$
J: P(\varepsilon, \bar{\sigma}, \bar{\alpha}) \longrightarrow P(\varepsilon, \bar{\sigma}, \bar{\alpha})
$$

is a continuous map.

This implies theorem 1.1. Since $P(\varepsilon, \bar{\sigma}, \bar{\alpha})$ is a compact subset in $C_{0}^{3, \alpha^{\prime}}\left(M^{\varepsilon}\right)$ for any $\alpha^{\prime}<\bar{\alpha}$ and $J: D \subset C_{0}^{3, \alpha^{\prime}}\left(M^{\varepsilon}\right) \longrightarrow C_{0}^{3, \alpha^{\prime}}\left(M^{\varepsilon}\right)$ is continuous, it must have a fixed point $u \in P(\varepsilon, \bar{\sigma}, \bar{\alpha})$.

Now for $u \in P(\varepsilon, \sigma, \alpha), J u=v+w$ where $v$ and $w$ are solutions of the linear equations:

$$
\begin{aligned}
L v & =-(K-1) \\
L w & =-E(u) .
\end{aligned}
$$

Thus proving theorem 2.1 involves estimating $v$ and $w$ in terms of $\varepsilon$ in various norms (we must show that $v+w \in P(\varepsilon, \sigma, \alpha)$ if $u \in P(\varepsilon, \sigma, \alpha)$ ).

Since $(K-1)$ does not depend on $u$, estimates of $v$ gives bounds on $P(\varepsilon, \sigma, \alpha)$ and smallness of $|K-1|$ in norms $L^{1}, L^{2}, C^{0, \alpha}$ assure that $v$ will be small in norms $C^{1, \alpha}, C^{0}$ and $H^{2}$. Recall that $|K-1|$ is a function depending on the construction of the approximate solution $M^{\varepsilon}$ (see section 3).

We estimate the $C^{0}$ norm of $v$ by a Sobolev inequality (see section 4 ) and by standard elliptic theory (see corollary 6.1). We use a Schauder estimate (see lemma 5.2) and the previous $C^{0}$ bound of $v$ to obtain the $C^{2, \alpha}$ norm of $v$.

There can be some explosion of the $C^{2, \alpha}$ norm since the constant of the Schauder estimate behaves like $\varepsilon^{-2}$ (see section 5); this is a consequence of the width of the strip and the fact that the Schauder estimates are local.

Interpolation inequalities (see lemma 5.1) give the $C^{1, \alpha}$ norm.

Then we can hope that $\mathrm{E}(\mathrm{u})$ will be smaller than $|K-1|$ in norm $L^{1}, L^{2}$ and $C^{0, \alpha}$ (see lemma 7.1) to obtain a better estimate for $w$ (see the beginning of the section 7).

In the case of $\mathrm{H}$-surfaces $\mathrm{N}$.Smale constructs a family of approximate solutions and a set $P(\varepsilon, \sigma, \alpha)$ such that $v \in P(\varepsilon, \sigma, \alpha)$ and $u \in P(\varepsilon, \sigma, \alpha)$ implies that $|u|_{C^{0}} \leq \varepsilon^{1,5-\sigma},|u|_{C^{2, \alpha}} \leq \varepsilon^{-0,5-\alpha-\sigma}$ and $|u|_{C^{1, \alpha}} \leq c \varepsilon^{0,5-\alpha-\sigma}$. 
In his lemma 6 , under the hypothesis $|u|<1,|\nabla u|<1$ he gets for a constant $C>0$ :

$$
|E(u)| \leq C\left(\eta|u|^{2}+|\nabla u|^{2}+\eta|u||\nabla u|+|u|\left|D^{2} u\right|+|\nabla u|^{2}\left|D^{2} u\right|\right) \text { (pointwise) }
$$

where $\eta$ is a regular function on $M^{\varepsilon}$, such that $|\eta|_{C^{0}} \leq c \varepsilon^{-1}$. But $\eta$ appears in the particular construction of the approximate solutions of N.Smale. Since terms $\left|D^{2} u\right|$ which behaves like $\varepsilon^{-0.5}$ are coupled with $|u|$ and $|\nabla u|^{2},|E(u)|$ will be small enough $\left(|E(u)|_{C^{0}} \leq c \varepsilon^{0,5-3 \sigma}\right)$ to obtain a good estimate for $w$.

In the case of $\mathrm{K}$-surfaces, under the hypothesis $|u|<1,|\nabla u|<1$ and $\left|D^{2} u\right|<1$, we obtain in lemma 7.1:

$$
|E(u)| \leq C\left(|u|^{2}+|\nabla u|^{2}+\left|D^{2} u\right|^{2}\right) \text { (pointwise). }
$$

We need $|u|_{C^{2, \alpha}}$ small enough to have a good estimate of $|E(u)|_{C^{0, \alpha}}\left(|u|_{C^{2, \alpha}}\right.$ can't behave like $\varepsilon^{-0,5}$ ).

$|K-1|_{C^{0}} \leq c \varepsilon^{2}$ implies that $|v|_{C^{0}} \leq c \varepsilon^{2,75}$ (see (3.13) and section 7(V1)) and thus $|v|_{C^{2, \alpha}} \leq c \varepsilon^{0.75-\alpha}$ (see lemma 5.2)and $|v|_{C^{3, \alpha}} \leq c \varepsilon^{-0.25-\alpha}$ (see lemma 5.4).

If $u \in P(\varepsilon, \sigma, \alpha)$, interpolation inequalities (lemma 5.3) gives that $|u|_{C^{2}} \leq$ $c \varepsilon^{0.75-\sigma}$ and $\mathrm{E}(\mathrm{u})$ will be small enough (see section 7 ).

To obtain $C^{0}$ bounds of $v, w$ we use $L^{1}$ and $L^{2}$ norms of $(K-1)$ and $E(u)$. Since $|E(u)|_{L^{1}} \leq c|u|_{H^{2}}^{2}$ and $|E(u)|_{L^{2}} \leq c|u|_{C^{2}}|u|_{H^{2}}$, we define $P(\varepsilon, \sigma, \alpha)$ with a $H^{2}$ bound. We use that $E(u) \in C^{1, \alpha}\left(M^{\varepsilon}\right)$ to get a bound of $|w|_{H^{2}}$. For these reasons we apply the Schauder fixed point theorem in $C_{0}^{3, \alpha}$ and we construct and attach the strip carefully in the section 3 .

In section 4,5,6 we prove some technical lemmas which allow us to estimate solutions of $L u=F, F \in C^{1, \alpha}\left(M^{\varepsilon}\right)$ as well as the eigenvalue bounds. Next, we use these lemmas to estimate $v$ and $w$ in section 7 .

\section{Construction of approximate solutions.}

Let $q_{1}$ and $q_{2}$ be points of $\partial S_{1}$ and $\partial S_{2}$. We extend $\left(S_{i}\right)_{i=1,2}$ to $\left(S_{i}^{\prime}\right)_{i=1,2}$ an open neighborhood of $\left(q_{i}\right)_{i=1,2}$ with $K=1>0$ by analyticity of the constant Gauss curvature surfaces.

Let $e_{1}, e_{2}$ be unit tangent vectors of $S_{1}^{\prime}, S_{2}^{\prime}$ at $q_{1}$ and $q_{2}$, orthogonal to $\partial S_{1}, \partial S_{2}$.

Let $P_{1}=\left(N_{q_{1}}, e_{1}\right)$ and $P_{2}=\left(N_{q_{2}}, e_{2}\right)$ be planes of $\mathbb{R}^{3}$, and let $B_{1}, B_{2}$ be spheres of radius 1 with their centers respectively at $P_{1}$ and $P_{2}$, such that $\left(B_{i}\right)_{i=1,2} \cap\left(S_{1} \cup S_{2}\right)=\emptyset .\left(B_{i}\right)_{i=1,2}$ are K-surfaces with $K=1$.

Lut $\gamma(t)$ be a smooth embedded path parametrized by arclength on $\left[0, l_{0}\right]$ such that: 
1. $\gamma: I=\left[0, l_{0}\right] \longrightarrow \mathbb{R}^{3}$ with $\gamma(0)=q_{1}, \gamma\left(l_{0}\right)=q_{2}$ and $\gamma \cap\left(S_{1} \cup S_{2}\right)=$ $\left\{q_{1}, q_{2}\right\}$ for $t \in\left[0, l_{0}\right]$.

2. $\gamma^{\prime}(0)=e_{1}, \gamma^{\prime}\left(l_{0}\right)=e_{2}$.

3. $0<\left|\gamma^{\prime \prime}(t)\right|$.

4. There exists $t_{1}, t_{2}, t_{3}, t_{4}$ some values in $\left[0, l_{0}\right]$ and $r_{0}>0, r_{1}>0$ small enough, such that

- $\forall t \in\left[0,2 r_{0}\right], \gamma(t) \subset P_{1} \cap S_{1}^{\prime}$

- $\forall t \in\left[2 r_{0}, t_{1}\right], \gamma(t) \subset P_{1}$

- $\forall t \in\left[t_{1}-r_{1}, t_{2}+r_{1}\right], \gamma(t) \subset B_{1}$

- $\forall t \in\left[t_{2}, t_{3}\right], \gamma(t)$ is a planar curve.

- $\forall t \in\left[t_{3}-r_{1}, t_{4}+r_{1}\right], \gamma(t) \subset B_{2}$

- $\forall t \in\left[t_{4}, l_{0}-2 r_{0}\right], \gamma(t) \subset P_{2}$

- $\forall t \in\left[l_{0}-2 r_{0}, l_{0}\right], \gamma(t) \subset P_{2} \cap S_{2}^{\prime}$.

We note:

$$
\begin{aligned}
n(t) & =\frac{\gamma^{\prime \prime}(t)}{\left|\gamma^{\prime \prime}(t)\right|} \\
b(t) & =\gamma^{\prime}(t) \wedge n(t) \\
\gamma^{\prime \prime}(t) & =k(t) n(t)
\end{aligned}
$$

with $k(t) \neq 0$ by 3$)$. When the curve is plane, we have $n^{\prime}(t)=-k(t) \gamma^{\prime}(t)$ and $\mathrm{b}(\mathrm{t})$ a constant vector.

Let $\mathrm{T}$ be any tubular neighborhood of $\gamma$. We construct a strip as a $C^{4, \beta}$ foliation of curves along $\gamma$ in T.

Let $P_{t}=N_{\gamma(t)} \gamma(t)$ the normal 2-dimensional space to $\gamma^{\prime}(t)$ at $\gamma(t)$, with the orthonormal frame $(\gamma(t), n(t), b(t))$. Then for each $\mathrm{t}$, we construct a piece of curve $C_{t}$ passing through $\gamma(t)$ in $P_{t}$ parametrized by arclength on $\left[-r_{2}, r_{2}\right]$ with $C_{t}(0)=0$ where $r_{2}>0$ is small enough such that $C_{t} \subset T$.

Then, let $x, y$ be functions $C^{4, \beta}$ on $\left[0, l_{0}\right] \times\left[-r_{2}, r_{2}\right]$ such that for $\mathrm{t}$ fixed and $s \in\left[-r_{2}, r_{2}\right], C_{t}(s)=x(t, s) n(t)+y(t, s) b(t)$.

We consider the $C^{4, \beta}$ surface $S_{r_{2}}$ parametrized by:

$$
\begin{aligned}
F_{r_{2}}:\left[0, l_{0}\right] \times\left[-r_{2}, r_{2}\right] & \longrightarrow \mathbb{R}^{3} \\
(t, s) & \longrightarrow \gamma(t)+x(t, s) n(t)+y(t, s) b(t)
\end{aligned}
$$


with $F_{r_{2}}(t, 0)=\gamma(t)$. Let $\mathrm{N}(\mathrm{t})$ be the normal vector to $S_{r_{2}}$ on $\gamma(t)$. Then

$$
N(t)=\frac{F_{t}(t, 0) \wedge F_{s}(t, 0)}{\left|F_{t}(t, 0) \wedge F_{s}(t, 0)\right|} .
$$

Let $\mathrm{K}(\mathrm{t}, \mathrm{s})$ be the Gauss curvature of $S_{r_{2}}$ at $F_{r_{2}}(t, s)$ and $K \in C^{2, \beta}\left(S_{r_{2}}, \mathbb{R}\right)$. We will need an estimate of $|K-1|_{C^{0}\left(S_{r_{2}}\right)}$ in terms of $r_{2}$.

Since

$$
K(t, s)=K(t, 0)+s K_{s}(t, 0)+\int_{0}^{s}(s-h) \frac{d^{2} K}{d h^{2}}(t, h s) d h ;
$$

if $K(t, 0)=1$ and $K_{s}(t, 0)=0$ we have

$$
|K-1|_{C^{0}} \leq\left|\frac{d^{2} K}{d s^{2}}\right|_{C^{0}} r_{2}^{2} .
$$

In the following lemma we study, conditions on $x, y$ to have $K(t, 0)=1$ and $K_{s}(t, 0)=0$. For $r_{2}>0$ small enough, $S_{r_{2}}$ will be strictly locally convex $(K>0)$. Then we construct a bridge in $\mathrm{T}$ which satisfy these conditions.

Lemma 3.1. Let $S$ be a compact surface in $\mathbb{R}^{3}, C^{4, \beta}$ up to the boundary, strictly locally convex and let $\gamma$ be a planar embedded curve of $S$ parametrized by arclength of class $C^{4, \beta}$

$$
\gamma(t):\left[0, r_{0}\right] \longrightarrow \mathbb{R}^{3}
$$

Assume also that the unit normal vector to $S$ at $\gamma(t)$ is

$$
N(t)=\cos \alpha(t) n(t)+\sin \alpha(t) b(t)
$$

where $\alpha(t)$ is a $C^{3, \beta}$ function

$$
\alpha(t):\left[0, r_{0}\right] \longrightarrow \mathbb{R}
$$

with $0 \leq \alpha(t) \leq \frac{\pi}{4}$.

We can locally parametrize the surface $S$ for $r_{0}>0$ small enough by

$$
\begin{aligned}
G:\left[0, r_{0}\right] \times\left[-r_{0}, r_{0}\right] & \longrightarrow \mathbb{R}^{3} \\
(t, s) & \longrightarrow \gamma(t)+X(t, s) n(t)+Y(t, s) b(t) .
\end{aligned}
$$

Where $X, Y$ are $C^{4, \beta}$ functions on $\left[0, r_{0}\right] \times\left[-r_{0}, r_{0}\right]$ such that the curve $C_{t}(s)=X(t, s) n(t)+Y(t, s) b(t)$ is parametrized by arclength with $C_{t}(0)=0$.

Let $K(t, s)$ be the Gauss curvature of $S$ at $G(t, s)$.

In the following we note $f$ for $f(t)$, a function which depends only on $t$, and its derivative $f$ '. 
1. If $S$ is a K-surface with $K=1$, then with $R=\frac{k \cos \alpha}{1+\left(\alpha^{\prime}\right)^{2}}$ we have:

(a)

$$
X(t, 0)=Y(t, 0)=0
$$

(b)

$$
X_{t}(t, 0)=Y_{t}(t, 0)=0
$$

(c)

$$
\begin{aligned}
& X_{s}(t, 0)=\sin \alpha \\
& Y_{s}(t, 0)=-\cos \alpha
\end{aligned}
$$

$$
X_{t t}(t, 0)=Y_{t t}(t, 0)=0
$$

(e)

$$
\begin{aligned}
& X_{s s}(t, 0)=\frac{1}{R} \cos \alpha \\
& Y_{s s}(t, 0)=\frac{1}{R} \sin \alpha
\end{aligned}
$$

(f)

$$
\begin{gathered}
X_{s t}(t, 0)=\alpha^{\prime} \cos \alpha \\
Y_{s t}(t, 0)=\alpha^{\prime} \sin \alpha
\end{gathered}
$$

(g)

$$
X_{t t t}(t, 0)=Y_{t t t}(t, 0)=0
$$

(h)

$$
\begin{aligned}
X_{t t s}(t, 0) & =\alpha^{\prime \prime} \cos \alpha-\left(\alpha^{\prime}\right)^{2} \sin \alpha \\
Y_{t t s}(t, 0) & =\alpha^{\prime \prime} \sin \alpha+\left(\alpha^{\prime}\right)^{2} \cos \alpha
\end{aligned}
$$

(i)

$$
\begin{aligned}
& X_{s s t}(t, 0)=-\frac{R^{\prime}}{R^{2}} \cos \alpha-\frac{\alpha^{\prime}}{R} \sin \alpha \\
& Y_{s s t}(t, 0)=-\frac{R^{\prime}}{R^{2}} \sin \alpha+\frac{\alpha^{\prime}}{R} \cos \alpha
\end{aligned}
$$

$$
X_{s s s}(t, 0)=\frac{3 k}{R} \sin \alpha \cos \alpha-\frac{\alpha^{\prime \prime}}{k R}-\frac{2 \alpha^{\prime} R^{\prime}}{k R^{2}}-4 \sin \alpha
$$

(j)

$$
\begin{aligned}
Y_{s s s}(t, 0)= & \frac{1}{R^{2} \cos \alpha}+\frac{3 k}{R} \sin ^{2} \alpha-\frac{\alpha^{\prime \prime} \sin \alpha}{k R \cos \alpha} \\
& -\frac{2 \alpha^{\prime} R^{\prime} \sin \alpha}{k R^{2} \cos \alpha}-\frac{4 \sin ^{2} \alpha}{\cos \alpha}
\end{aligned}
$$


Bridge principle for constant and positive Gauss curvature surfaces 513

2. Reciprocally if $S$ is a surface with Gauss curvature $K$ not necessarily constant and if $X, Y$ verify a)-j), with $N$ as above then $K(t, 0)=1$ and $K_{s}(t, 0)=0$ for $t \in\left[0, r_{0}\right]$.

Proof. 1)

- $C_{t}(0)=0 \Rightarrow a$ ) and $a$ ) implies b), d), g).

- $C_{t}(s)$ parametrized by arclength implies $X_{s}^{2}+Y_{s}^{2}=1$ and

$$
G_{s}(t, 0)=X_{s} n+Y_{s} b
$$

orthogonal to $\mathrm{N}(\mathrm{t})$ implies $\mathrm{c}$ ).

- $c) \Rightarrow f) \Rightarrow h)$.

- $e) \Rightarrow i)$.

Then we have to prove e) and $\mathrm{j}$ ).

Since

$$
X_{s}^{2}+Y_{s}^{2}=1
$$

we have on $\mathrm{S}$

$$
X_{s} X_{s s}+Y_{s} Y_{s s}=0 \text { and } X_{s s}^{2}+Y_{s s}^{2}+X_{s} X_{s s s}+Y_{s} Y_{s s s}=0,
$$

which implies by c) for each point $(t, 0)$ :

$$
X_{s s} \sin \alpha-Y_{s s} \cos \alpha=0
$$

and by a), b), c), d), f) and e) for each point (t,0):

$$
\frac{1}{R^{2}}+X_{s s s} \sin \alpha-Y_{s s s} \cos \alpha=0 \text {. }
$$

We write $\mathrm{K}(\mathrm{t}, \mathrm{s})$ in terms of $\mathrm{X}, \mathrm{Y}, \mathrm{DX}, \mathrm{DY}, D^{2} X, D^{2} Y$ and by a), b), c), d), f) we have:

$$
K(t, 0)=k \cos \alpha\left(X_{s s} \cos \alpha+Y_{s s} \sin \alpha\right)-\left(\alpha^{\prime}\right)^{2}=1
$$

which gives e) with (3.2). Now by e) we have (3.3).

Since $\mathrm{S}$ is a $\mathrm{K}$-surface, we have $K_{s}(t, s)=0$. As above we write $K_{s}(t, 0)$ in terms of $\mathrm{X}, \mathrm{Y}, \mathrm{DX}, \mathrm{DY}, D^{2} X, D^{2} Y, D^{3} X, D^{3} Y$, and by a)-i) we have:

$$
\begin{aligned}
& K_{s}(t, 0)=\frac{\alpha^{\prime \prime}}{R}-\frac{2 k^{2}}{R} \cos \alpha \sin \alpha-\frac{k}{R^{2}} \sin \alpha+ \\
& \quad k \cos \alpha\left(X_{s s s} \cos \alpha+Y_{s s s} \sin \alpha-\frac{k}{R} \sin \alpha\right)+\frac{2 \alpha^{\prime} R^{\prime}}{R^{2}}+4 k \sin \alpha=0
\end{aligned}
$$


which gives us $\mathrm{j}$ ) with (3.3).

- proof of e).

By direct computation with $\gamma^{\prime \prime}=k n, n^{\prime}=-k \gamma^{\prime}(k>0$ since S is strictly locally convex) and $b^{\prime}=0$ (since $\gamma$ is a planar curve ):

$$
\begin{aligned}
G_{t}(t, s) & =(1-k X) \gamma^{\prime}+X_{t} n+Y_{t} b \\
G_{s}(t, s) & =X_{s} n+Y_{s} b \\
G_{t} \wedge G_{s}(t, s) & =\left(X_{t} Y_{s}-Y_{t} X_{s}\right) \gamma^{\prime}-Y_{s}(1-k X) n+X_{s}(1-k X) b \\
G_{t t}(t, s) & =-\left(k_{t} X+2 k X_{t}\right) \gamma^{\prime}+\left(k(1-k X)+X_{t t}\right) n+Y_{t t} b \\
G_{s s}(t, s) & =X_{s s} n+Y_{s s} b \\
G_{s t}(t, s) & =-k X_{s} \gamma^{\prime}+X_{s t} n+Y_{s t} b \\
g_{11}(t, s) & =\left\langle G_{t}, G_{t}\right\rangle=(1-k X)^{2}+X_{t}^{2}+Y_{t}^{2} \\
g_{22}(t, s) & =\left\langle G_{s}, G_{s}\right\rangle=X_{s}^{2}+Y_{s}^{2} \\
g_{12}(t, s) & =\left\langle G_{t}, G_{s}\right\rangle=X_{t} X_{s}+Y_{t} Y_{s}
\end{aligned}
$$

and

$$
K(t, s)=\frac{e g-f^{2}}{\left(g_{11} g_{22}-g_{12}^{2}\right)^{2}}
$$

(see $[\mathrm{DoC}])$ since $\left|G_{t} \wedge G_{s}\right|=\sqrt{g_{11} g_{22}-g_{12}^{2}}$ with

$$
\begin{aligned}
e(t, s)= & \left\langle G_{t} \wedge G_{s}, G_{t t}\right\rangle=-\left(k^{\prime} X+2 k X_{t}\right)\left(X_{t} Y_{s}-Y_{t} X_{s}\right) \\
& -Y_{s}(1-k X)\left(k(1-k X)+X_{t t}\right)+X_{s} Y_{t t}(1-k X) \\
g(t, s)= & \left\langle G_{t} \wedge G_{s}, G_{s s}\right\rangle \\
= & X_{s} Y_{s s}(1-k X)-Y_{s} X_{s s}(1-k X) \\
f(t, s)= & \left\langle G_{t} \wedge G_{s}, G_{s t}\right\rangle=-k X_{s}\left(X_{t} Y_{s}-Y_{t} X_{s}\right) \\
& -Y_{s} X_{s t}(1-k X)+X_{s} Y_{s t}(1-k X)
\end{aligned}
$$

Then by a), b) c),d), f), we have:

$$
\begin{aligned}
g_{11}(t, 0) & =1 \\
g_{22}(t, 0) & =1 \\
g_{12}(t, 0) & =0 \\
e(t, 0) & =k \cos \alpha \\
g(t, 0) & =X_{s s} \cos \alpha+Y_{s s} \sin \alpha \\
f(t, 0) & =\alpha^{\prime}
\end{aligned}
$$


Bridge principle for constant and positive Gauss curvature surfaces 515

Thus (3.4):

$$
K(t, 0)=k \cos \alpha\left(X_{s s} \cos \alpha+Y_{s s} \sin \alpha\right)-\left(\alpha^{\prime}\right)^{2} .
$$

Then with (3.2) we have the following system:

$$
\left\{\begin{array}{l}
X_{s s} \sin \alpha-Y_{s s} \cos \alpha=0 \\
k \cos \alpha\left(X_{s s} \cos \alpha+Y_{s s} \sin \alpha\right)-\left(\alpha^{\prime}\right)^{2}=1
\end{array}\right.
$$

Thus:

which gives us e).

$$
\left\{\begin{array}{l}
Y_{s s}=X_{s s} \frac{\sin \alpha}{\cos \alpha} \\
X_{s s}=\frac{1+\left(\alpha^{\prime}\right)^{2}}{k \cos \alpha} \cos \alpha
\end{array}\right.
$$

- proof of $\mathrm{j}$ ).

By derivation in s of (3.6) we have:

$$
\begin{aligned}
& g_{11 s}(t, s)=-2 k X_{s}(1-k X)+2 X_{t s} X_{t}+2 Y_{t s} Y_{t} \\
& g_{22 s}(t, s)=0 \\
& g_{12 s}(t, s)=X_{t s} X_{s}+X_{t} X_{s s}+Y_{t s} Y_{s}+Y_{t} Y_{s s}
\end{aligned}
$$

and by a)-f):

$$
\begin{aligned}
& g_{11 s}(t, 0)=-2 k \sin \alpha \\
& g_{22 s}(t, 0)=0 \\
& g_{12 s}(t, 0)=0
\end{aligned}
$$

By derivation in s of (3.8) we have:

$$
\begin{aligned}
e_{s}(t, s)= & -\left(k^{\prime} X+2 k X_{t}\right)_{s}\left(X_{t} Y_{s}-Y_{t} X_{s}\right)-\left(k^{\prime} X+2 k X_{t}\right)\left(X_{t} Y_{s}-Y_{t} X_{s}\right)_{s} \\
& -Y_{s s}(1-k X)\left(k(1-k X)+X_{t t}\right) \\
& +k Y_{s} X_{s}\left(k(1-k X)+X_{t t}\right)-Y_{s}(1-k X)\left(-k^{2} X_{s}+X_{t t s}\right) \\
& +X_{s s} Y_{t t}(1-k X)+X_{s} Y_{t t s}(1-k X)-k X_{s}^{2} Y_{t t} \\
g_{s}(t, s)= & (1-k X)\left(X_{s} Y_{s s s}-Y_{s} X_{s s s}\right)+k X_{s}\left(Y_{s} X_{s s}-X_{s} Y_{s s}\right) \\
f_{s}(t, s)= & -k X_{s s}\left(X_{t} Y_{s}-Y_{t} X_{s}\right)-k X_{s}\left(X_{t} Y_{s s}-Y_{t} X_{s s}\right) \\
& +(1-k X)\left(X_{s s} Y_{s t}+X_{s} Y_{s t s}-Y_{s s} X_{s t}-Y_{s} X_{s t s}\right)
\end{aligned}
$$


and by a)-f):

$$
\begin{aligned}
e_{s}(t, 0) & =2 k^{2} X_{s} Y_{s}-k Y_{s s}-Y_{s} X_{t t s}+X_{s} Y_{t t s} \\
& =\alpha^{\prime \prime}-2 k^{2} \cos \alpha \sin \alpha-\frac{k}{R} \sin \alpha \\
g_{s}(t, 0) & =X_{s s s} \cos \alpha+Y_{s s s} \sin \alpha-\frac{k}{R} \sin \alpha \\
f_{s}(t, 0) & =\left(X_{s s} Y_{s t}+X_{s} Y_{s t s}-Y_{s s} X_{s t}-Y_{s} X_{s t s}\right)=-\frac{R^{\prime}}{R^{2}}
\end{aligned}
$$

By derivation in s of (3.7) we have:

$$
K_{s}(t, 0)=\left(e_{s} g+e g_{s}-2 f f_{s}\right)-2\left(g_{11} g_{22}-g_{12}^{2}\right)_{s}\left(e g-f^{2}\right) .
$$

Then:

$$
\begin{aligned}
K_{s}(t, 0)= & \frac{\alpha^{\prime \prime}}{R}-\frac{2 k^{2}}{R} \cos \alpha \sin \alpha-\frac{k}{R^{2}} \sin \alpha \\
& +k \cos \alpha\left(X_{s s s} \cos \alpha+Y_{s s s} \sin \alpha-\frac{k}{R} \sin \alpha\right) \\
& +\frac{2 \alpha^{\prime} R^{\prime}}{R^{2}}+4 k \sin \alpha .
\end{aligned}
$$

Thus with (3.3) we get the following system:

$$
\left\{\begin{aligned}
X_{s s s} \cos \alpha+Y_{s s s} \sin \alpha= & -\frac{\alpha^{\prime \prime}}{k R \cos \alpha}+\frac{3 k}{R} \sin \alpha+\frac{\sin \alpha}{R^{2} \cos \alpha} \\
& -\frac{2 \alpha^{\prime} R^{\prime}}{k R^{2} \cos \alpha}-\frac{4 \sin \alpha}{\cos \alpha} \\
\frac{1}{R^{2}}+X_{s s s} \sin \alpha-Y_{s s s} \cos \alpha & =0
\end{aligned}\right.
$$

and

$$
\left\{\begin{array}{l}
Y_{s s s}=\frac{1}{R^{2} \cos \alpha}+X_{s s s} \frac{\sin \alpha}{\cos \alpha} \\
X_{s s s}=\frac{3 k}{R} \sin \alpha \cos \alpha-\frac{\alpha^{\prime \prime}}{k R}-\frac{2 \alpha^{\prime} R^{\prime}}{k R^{2}}-4 \sin \alpha
\end{array}\right.
$$

gives $\mathrm{j}$ ).

2) Reciprocally, $\mathrm{K}(\mathrm{t}, 0)$ and $K_{s}(t, 0)$ depending only of a)-j), $\mathrm{k}, \mathrm{k}$, the calculus of (3.4), (3.5) above is true.

Now we construct the bridge $S_{r_{2}}$ by piecewise. 
1) For $t \in I_{1}=\left[0, t_{1}\right] \cup\left[t_{2}, t_{3}\right] \cup\left[t_{4}, l_{0}\right], \gamma$ is a planar curve. For $s \in\left[-r_{0}, r_{0}\right]$ we define $C_{t}(s)=x(t, s) n+y(t, s) b$ with $R=k$ by:

$$
\begin{aligned}
& x(t, s)=R\left(1-\cos \frac{s}{R}\right) \\
& y(t, s)=-R \sin \left(\frac{s}{R}\right) .
\end{aligned}
$$

We consider the embedding:

$$
\begin{aligned}
F_{1}: I_{1} \times\left[-r_{2}, r_{2}\right] & \longrightarrow \mathbb{R}^{3} \\
(t, s) & \longrightarrow \gamma+x(t, s) n+y(t, s) b
\end{aligned}
$$

Since $\gamma$ is a smooth curve, $F_{1}$ is a $C^{4, \beta}$ embedding of $I_{1} \times\left[-r_{2}, r_{2}\right]$ for $r_{2}>0$ small enough, and $\left|F_{1}\right|_{C^{4, \beta}} \leq C_{0},\left|D F_{1}\right| \geq C_{0}^{-1}$ for $C_{0}>0$ large enough independent of $r_{2}$. Moreover $F_{1}$ verifies lemma 3.1-2).

We have:

$$
x(t, 0)=y(t, 0)=0
$$

and $a$ ) implies $b), d), g$ ) of lemma 3.1.

$$
x_{s}(t, s)=\sin \frac{s}{R} \text { and } y_{s}(t, s)=-\cos \frac{s}{R}
$$

implies

$$
x_{s}(t, 0)=0 \text { and } y_{s}(t, 0)=-1 \text {. }
$$

Then

$$
F_{t}(t, 0)=\gamma^{\prime} \text { and } F_{s}(t, 0)=-b
$$

which implies $N(t)=n$.

Then $x, y$ verify a)-j) of lemma 3.1 with $\alpha=\alpha^{\prime}=\alpha^{\prime \prime}=0$ and $R=k$ for $t \in I_{1}$ : we have a), b), c), d), g) and $\left.c\right) \Rightarrow f(\Rightarrow h)$.

$$
x_{s s}(t, s)=\frac{1}{R} \cos \frac{s}{R} \text { and } y_{s s}(t, s)=\frac{1}{R} \sin \frac{s}{R}
$$

implies

$$
x_{s s}(t, 0)=\frac{1}{R} \text { and } y_{s s}(t, 0)=0
$$

e) implies i).

$$
x_{s s s}(t, s)=-\frac{1}{R^{2}} \sin \frac{s}{R} \text { and } y_{s s s}(t, s)=\frac{1}{R^{2}} \cos \frac{s}{R}
$$


implies

$$
x_{s s s}(t, s)=0 \text { and } y_{s s s}(t, s)=\frac{1}{R^{2}}
$$

Then for $t \in I_{1}$, we have $K(t, 0)=1$ and $K_{s}(t, 0)=0$ by lemma 3.1 .

2) For $t \in\left[t_{1}-r_{0}, t_{2}+r_{0}\right] \cup\left[t_{3}-r_{0}, t_{4}+r_{0}\right], \gamma(t)$ is a curve of the K-surface $B_{1} \cup B_{2}$, with $K=1$. Then we take $C_{t}$ parametrized as above of length $2 r_{2}$ such that $C_{t} \subset P_{t} \cap\left(B_{1} \cup B_{2}\right)$ and $C_{t}(0)=0$. Then

$$
\begin{aligned}
F_{2}: I_{2} \times\left[-r_{2}, r_{2}\right] & \longrightarrow \mathbb{R}^{3} \\
(t, s) & \longrightarrow \gamma+x(t, s) n+y(t, s) b
\end{aligned}
$$

is a $C^{4, \beta}$ embedded for $r_{2}>0$ small enough and

$$
F_{2}\left(I_{2} \times\left[-r_{2}, r_{2}\right]\right) \subset B_{1} \cup B_{2} .
$$

Then

$$
K(t, s)=1 \text { for } t \in I_{2}
$$

Let

$$
F_{r_{2}}=\left\{\begin{array}{l}
F_{1} \text { if }(t, s) \in I_{1} \times\left[-r_{2}, r_{2}\right] \\
F_{2} \text { if }(t, s) \in I_{2} \times\left[-r_{2}, r_{2}\right]
\end{array}\right.
$$

Since for $t \in I_{1} \cap I_{2}, \gamma$ is a piece of great circle of the spheres $B_{1}$ or $B_{2}$ (Their centers are in the plan $P_{1}, P_{2}$ ), then $F_{1}=F_{2}$ for $t \in I_{1} \cap I_{2}$ and $F_{r_{2}}$ is a $C^{4, \beta}$ embedding of $\left[0, l_{0}\right] \times\left[-r_{2}, r_{2}\right]$.

Clearly $\left|F_{r_{2}}\right|_{C^{4, \beta}} \leq C_{0}$ and $\left|D F_{r_{2}}\right|>C_{0}^{-1}$ for $C_{0}>0$ large enough.

Now we smooth out the embedding in small neighborhood of $q_{1}$ and $q_{2}$, so that the strip is smoothly attached to $S_{1} \cup S_{2}$.

Let $F_{\varepsilon}$ be the restriction of $F_{r_{2}}=F$ :

$$
F_{\varepsilon}=F_{\mid\left[2 r_{0}, l_{0}-2 r_{0}\right] \times[-\varepsilon, \varepsilon]} \text { with } 0<r_{0}<1 \text { small enough. }
$$

We claim that we can extend $F_{\varepsilon}$ to $[-\varepsilon, \varepsilon] \times\left[0, l_{0}\right]$ so that it's image is a $C^{4, \beta}$ extension of $S_{1} \cup S_{2}$ and

$$
\left|F_{\varepsilon}\right|_{C^{4, \beta}} \leq C_{0}, \quad\left|D F_{\varepsilon}\right|>C_{0}^{-1}
$$

for $C_{0}>0$ large enough independent of $\varepsilon$.

3) For $t \in I_{3}=\left[0, r_{0}\right], \gamma$ is a planar curve of $S_{1}^{\prime}$. Let $N(t)=\cos \alpha n+\sin \alpha b$ be the normal vector on $S_{1}^{\prime}$ at $\gamma(t)$ defined on $\left[0,2 r_{0}\right]$. Then $\alpha(t):\left[0,2 r_{0}\right] \longrightarrow \mathbb{R}$ 
Bridge principle for constant and positive Gauss curvature surfaces 519 is an analytic map, since a K-surface is analytic in its' interior. Moreover $\alpha(0)=0$ implies $0 \leq \alpha \leq \pi / 4$ for $r_{0}>0$ small enough.

We can locally parametrize $S_{1}^{\prime}$ around $\gamma$ by:

$$
\begin{aligned}
G:\left[0,2 r_{0}\right] \times[-\varepsilon, \varepsilon] & \longrightarrow \mathbb{R}^{3} \\
(t . s) & \longrightarrow \gamma+X(t, s) n+Y(t, s) b
\end{aligned}
$$

where X,Y satisfy Lemma 3.1-1).

Let $x, y$ be functions defined on $\left[0,2 r_{0}\right] \times[-\varepsilon, \varepsilon]$ by:

$$
\begin{aligned}
& x(t, s)=R\left(\cos \alpha-\cos \left(\alpha+\frac{s}{R}\right)\right)+\frac{s^{3}}{6}\left(X_{s s s}(t, 0)+\frac{1}{R^{2}} \sin \alpha\right) \\
& y(t, s)=R\left(\sin \alpha-\sin \left(\alpha+\frac{s}{R}\right)\right)+\frac{s^{3}}{6}\left(Y_{s s s}(t, 0)-\frac{1}{R^{2}} \cos \alpha\right)
\end{aligned}
$$

with $R=\frac{k \cos \alpha}{1+\left(\alpha^{\prime}\right)^{2}}$ and $X_{s s s}(t, 0), Y_{s s s}(t, 0)$ defined in $\left.\mathrm{j}\right)$ of Lemma 3.1.

We claim that $x, y$ verifies a)-j) of lemma 3.1 :

Since

$$
x(t, 0)=y(t, 0)=0
$$

we have b), d), g).

$$
\begin{aligned}
& x_{s}(t, s)=\sin \left(\alpha+\frac{s}{R}\right)+\frac{s^{2}}{2}\left(X_{s s s}(t, 0)+\frac{1}{R^{2}} \sin \alpha\right) \\
& y_{s}(t, 0)=-\cos \left(\alpha+\frac{s}{R}\right)+\frac{s^{2}}{2}\left(Y_{s s s}(t, 0)-\frac{1}{R^{2}} \cos \alpha\right)
\end{aligned}
$$

implies

$$
x_{s}(t, 0)=\sin \alpha \text { and } y_{s}(t, 0)=-\cos \alpha
$$

and we have f),h).

$$
\begin{aligned}
& x_{s s}(t, s)=\frac{1}{R} \cos \left(\alpha+\frac{s}{R}\right)+s\left(X_{s s s}(t, 0)+\frac{1}{R^{2}} \sin \alpha\right) \\
& y_{s s}(t, s)=\frac{1}{R} \sin \left(\alpha+\frac{s}{R}\right)+s\left(Y_{s s s}(t, 0)-\frac{1}{R^{2}} \cos \alpha\right)
\end{aligned}
$$

implies

$$
x_{s s}(t, 0)=\frac{1}{R} \cos \alpha \text { and } y_{s s}(t, 0)=\frac{1}{R} \sin \alpha
$$


and we have i).

$$
\begin{aligned}
& x_{s s s}(t, s)=-\frac{1}{R^{2}} \sin \left(\alpha+\frac{s}{R}\right)+X_{s s s}(t, 0)+\frac{1}{R^{2}} \sin \alpha \\
& y_{s s s}(t, s)=\frac{1}{R^{2}} \cos \left(\alpha+\frac{s}{R}\right)+Y_{s s s}(t, 0)-\frac{1}{R^{2}} \cos \alpha
\end{aligned}
$$

implies

(e)

$$
x_{s s s}(t, 0)=X_{s s s}(t, 0) \text { and } y_{s s s}(t, 0)=Y_{s s s}(t, 0)
$$

Thus

$$
\begin{aligned}
x(t, 0) & =X(t, 0) & y(t, 0) & =Y(t, 0) \\
D x(t, 0) & =D X(t, 0) & D y(t, 0) & =D Y(t, 0) \\
D^{2} x(t, 0) & =D^{2} X(t, 0) & D^{2} y(t, 0) & =D^{2} Y(t, 0) \\
D^{3} x(t, 0) & =D^{3} X(t, 0) & D^{3} y(t, 0) & =D^{3} Y(t, 0)
\end{aligned}
$$

Let $\phi_{1}$ be a cutoff function on $\mathbb{R}$ such that:

$$
\begin{aligned}
& \phi_{1}: \mathbb{R} \longrightarrow \mathbb{R} \text { and } 0 \leq \phi_{1}(t) \leq 1 \\
& \phi_{1}(t)=1 \text { for } t \leq \frac{r_{0}}{2} \\
& \phi_{1}(t)=0 \text { for } t \geq \frac{3 r_{0}}{4}
\end{aligned}
$$

and $\max \left|D^{k} \phi_{1}\right| \leq c_{1},\left|D^{k} \phi_{1}\right|_{(\alpha)} \leq c_{1}$ for $\mathrm{k}=1,2,3,4$ and $0<\alpha<1$, for some constant $c_{1}$ independent of $\varepsilon$.

Let $F_{3}$ be the embedding of $\left[0, r_{0}\right] \times[-\varepsilon, \varepsilon]$ defined by:

$$
\begin{aligned}
F_{3}:\left[0, r_{0}\right] \times[-\varepsilon, \varepsilon] & \longrightarrow \mathbb{R}^{3} \\
(t, s) & \longrightarrow \gamma+\left(\phi_{1} X+\left(1-\phi_{1}\right) x\right) n+\left(\phi_{1} Y+\left(1-\phi_{1}\right) y\right) b .
\end{aligned}
$$

Then using a Taylor expansion for $x, y, X, Y$ in s, it's easy to see that $F_{3}$ verify the lemma 3.1 and:

$$
\begin{aligned}
\left|D F_{3}\right| & \geq C_{0}^{-1} \\
\left|F_{3}\right|_{C^{4, \beta}} & \leq C_{0} .
\end{aligned}
$$

4) For $t \in I_{4}=\left[r_{0}, 2 r_{0}\right], \gamma \in P_{1}=\left(N(0), e_{1}\right) \cap S_{1}^{\prime}$.

Let $\phi_{2}$ be a cutoff function on $\mathbb{R}$ such that:

$$
\begin{aligned}
& \phi_{2}: \mathbb{R} \longrightarrow \mathbb{R} \text { and } 0 \leq \phi_{2}(t) \leq 1 \\
& \phi_{2}(t)=1 \text { for } t \leq \frac{3 r_{0}}{2} \\
& \phi_{2}(t)=0 \text { for } t \geq \frac{7 r_{0}}{4}
\end{aligned}
$$


Bridge principle for constant and positive Gauss curvature surfaces 521

and $\max \left|D^{k} \phi_{2}\right| \leq c_{3},\left|D^{k} \phi_{2}\right|_{(\alpha)} \leq c_{3}$ for $\mathrm{k}=1,2,3,4$ and $0<\alpha<1$, for some constant $c_{3}$ independent of $\varepsilon$.

Let $F_{4}$ be the embedding of $\left[r_{0}, 2 r_{0}\right] \times[-\varepsilon, \varepsilon]$ defined by:

$$
\begin{aligned}
F_{4}:\left[r_{0}, 2 r_{0}\right] \times[-\varepsilon, \varepsilon] & \longrightarrow \mathbb{R}^{3} \\
(t, s) & \longrightarrow \gamma+x n+y b
\end{aligned}
$$

with

$$
\begin{aligned}
& x(t, s)=R\left(\cos \beta-\cos \left(\beta+\frac{s}{R}\right)\right)+\frac{s^{3}}{6}\left(f_{1}(t)+\frac{1}{R^{2}} \sin \beta\right) \\
& y(t, s)=R\left(\sin \beta-\sin \left(\beta+\frac{s}{R}\right)\right)+\frac{s^{3}}{6}\left(f_{2}(t)-\frac{1}{R^{2}} \cos \beta\right)
\end{aligned}
$$

where

$$
\begin{aligned}
\beta & =\phi_{2} \alpha \\
R & =\frac{k \cos \beta}{1+\left(\beta^{\prime}\right)^{2}} \\
f_{1}(t) & =\frac{3 k}{R} \sin \beta \cos \beta-\frac{\beta^{\prime \prime}}{k R}-\frac{2 \beta^{\prime} R_{t}}{k R^{2}}-4 \sin \beta \\
f_{2}(t) & =\frac{1}{R^{2} \cos \beta}+\frac{3 k}{R} \sin ^{2} \beta-\frac{\beta^{\prime \prime} \sin \beta}{k R \cos \beta}-\frac{2 \beta^{\prime} R_{t} \sin \beta}{k R^{2} \cos \beta}-\frac{4 \sin ^{2} \beta}{\cos \beta}
\end{aligned}
$$

Since $\alpha$ and $\phi_{2}$ are $C^{\infty}$, then $\beta$ is $C^{\infty}$ and $F_{4}$ is a $C^{4, \beta}$ embedding of $\left[r_{0}, 2 r_{0}\right] \times[-\varepsilon, \varepsilon]$ for $\varepsilon>0$ small enough. Moreover it's clear that $x, y$ verify a)-j) of lemma 3.1 with $N(t)=\cos \beta n+\sin \beta b$.

We define the $C^{4, \beta}$ extension of $F_{\varepsilon}$ near $q_{1}$ by:

$$
\Psi_{\varepsilon}=\left\{\begin{array}{l}
F_{1} \text { if }(t, s) \in\left(\left[2 r_{0}, t_{1}\right] \cup\left[t_{2}, t_{3}\right] \cup\left[t_{4}, l_{0}-2 r_{0}\right]\right) \times[-\varepsilon, \varepsilon] \\
F_{2} \text { if }(t, s) \in\left(\left[t_{1}, t_{2}\right] \cup\left[t_{3}, t_{4}\right]\right) \times[-\varepsilon, \varepsilon] \\
F_{3} \text { if }(t, s) \in\left[0, r_{0}\right] \times[-\varepsilon, \varepsilon] \\
F_{4} \text { if }(t, s) \in\left[r_{0}, 2 r_{0}\right] \times[-\varepsilon, \varepsilon]
\end{array}\right.
$$

Similarly, extending $F_{\varepsilon}$ near $q_{2}$, we get a $C^{4, \beta}$ embedding

$$
\Psi_{\varepsilon}:\left[0, l_{0}\right] \times[-\varepsilon, \varepsilon] \longrightarrow \mathbb{R}
$$

with $K(t, 0)=1$ and $K_{s}(t, 0)=0$, with the $C^{4, \beta}$ bound

$$
\left|D \Psi_{\varepsilon}\right| \geq C_{0}^{-1} \text { and }\left|\Psi_{\varepsilon}\right|_{C^{4, \beta}} \leq C_{0} .
$$


Now to construct a $C^{4, \beta}$ approximate solution $M^{\varepsilon}$ we need to move the boundary of the strip towards $\gamma$ in a sufficiently nice way:

Let $\mathrm{f}$ be a function on $[0, \infty$ [ whose graph is smooth such that:

$$
\left\{\begin{array}{l}
f(0)=1 \\
f(t)=\frac{1}{2} \text { for } t>\frac{1}{4} \\
f^{\prime}(t)<0 \text { for } 0<s<\frac{1}{4}
\end{array}\right.
$$

and the graph of $f$ is smoothly tangent to the $y$-axis.

Let

$$
f_{\varepsilon}(t)= \begin{cases}\varepsilon f\left(\frac{t}{\varepsilon}\right) & t \in\left[0, \frac{\varepsilon}{4}\right] \\ \frac{\varepsilon}{2} & t \in\left[\frac{\varepsilon}{4}, l_{0}-\frac{\varepsilon}{4}\right] \\ \varepsilon f\left(\frac{l_{0}-t}{\varepsilon}\right) & t \in\left[l_{0}-\frac{\varepsilon}{4}, l_{0}\right]\end{cases}
$$

Now we get a family of thin strips $S^{\varepsilon}$ defined by

$$
S^{\varepsilon}=\left\{(t, s) \in\left[0, l_{0}\right] \times[-\varepsilon, \varepsilon] /|s| \leq f_{\varepsilon}(t)\right\}
$$

and $M^{\varepsilon}=S_{1} \cup S_{2} \cup S^{\varepsilon}$. Thus $M^{\varepsilon}$ is a $C^{4, \beta}$ surface up to the boundary $\partial M^{\varepsilon}$, that is $S_{1}$ connected to $S_{2}$ by a strip of width $\varepsilon$ that contains $\gamma$, and is itself contained in T. We will often identify $S^{\varepsilon}$ with $\Psi_{\varepsilon}\left(S^{\varepsilon}\right) . M^{\varepsilon}$ is embedded if $S_{1}$ and $S_{2}$ are embedded.

Since $\Psi_{\varepsilon}$ parametrize the strip, we can find a finite collection of parametrizations of $M^{\varepsilon},\left(\left(\Omega_{j}, \Psi_{j}\right)\right)_{j=1 \ldots J}$ with $\Psi_{1}=\Psi_{\varepsilon}, \Omega_{1}=S^{\varepsilon}$ and $\Psi_{j}: \Omega_{j} \longrightarrow M^{\varepsilon}, i=1 \ldots J$ covering $S_{1} \cup S_{2}$.

Of course $C_{0}^{-1} \leq\left|D \Psi_{1}\right| \leq C_{0}$ so lengths and volumes are equivalent in $\Omega_{1}$ and $\Psi_{1}\left(\Omega_{1}\right)$. If $g_{i j}$ is the metric tensor induced by $\Psi_{1}$ and $\left(g^{i j}\right)=\left(g_{i j}\right)^{-1}$ then we have

$$
\begin{aligned}
& \sum_{i, j}\left(\left|g_{i j}\right|_{C^{3, \beta}\left(S^{\varepsilon}\right)}+\left|g^{i j}\right|_{C^{3, \beta}\left(S^{\varepsilon}\right)}\right) \leq C_{0} \\
& \lambda^{-1} I \leq\left(g_{i j}\right) \leq \lambda I \\
& \lambda^{-1} I \leq\left(g^{i j}\right) \leq \lambda I \\
& I=\text { identity matrix }
\end{aligned}
$$

For $C_{0}>0$ and $\lambda>0$ some constant independent of $\varepsilon . M^{\varepsilon}$ is an approximate solution in the sense that the Gauss curvature of $M^{\varepsilon}$ is near 1 in the norm 
$C^{1, \alpha} . K=1$ on $S_{1} \cup S_{2}$ and on $S^{\varepsilon}$ we have by construction and (3.1):

$$
K(t, s)=1+\int_{0}^{s}(s-h) \frac{d^{2} K}{d s^{2}}(t, h s) d h
$$

Since $\left|\frac{d^{2} K}{d s^{2}}\right|_{C^{0}\left(S^{\varepsilon}\right)}$ depends on a bound of $\Psi_{\varepsilon}$ in the norm $C^{4}$ we have:

$$
|K-1|_{C^{0}\left(M_{\varepsilon}\right)} \leq c_{1} \varepsilon^{2}
$$

Then

$$
\int_{M^{\varepsilon}}|K-1|^{p}=\int_{S_{\varepsilon}}|K-1|^{p} \leq c_{1} \varepsilon^{2 p} \operatorname{Vol}\left(S_{\varepsilon}\right) \leq c_{2} \varepsilon^{2 p} \varepsilon
$$

Thus

$$
\begin{aligned}
|K-1|_{L^{1}\left(M^{\varepsilon}\right)} & \leq c_{3} \varepsilon^{3} \\
|K-1|_{L^{2}\left(M^{\varepsilon}\right)} & \leq c_{3} \varepsilon^{2,5}
\end{aligned}
$$

Moreover since $K(t, 0)=1$ we have in the same way:

$$
|K-1|_{C^{1, \alpha}\left(M_{\varepsilon}\right)} \leq c_{4} \varepsilon^{1-\alpha} \text { and }|K-1|_{C^{1}\left(M^{\varepsilon}\right)} \leq c_{5} \varepsilon
$$

for some constants $c_{1}, c_{2}, c_{3}, c_{4}, c_{5}$ independent of $\varepsilon$.

\section{Technical lemma.}

As in [20], we prove a technical lemma to obtain a $C^{0}$ estimate. We use the same technique and obtain a $|u|_{L^{p}}$ terms to the right hand side since the mean curvature is only bounded.

Lemma 4.1. Let $\Gamma$ be any positive constant. Then for $u \in C^{2}\left(M^{\varepsilon}\right)$ with $u_{\mid \partial M^{\varepsilon}}$ identically zero and $|u|_{L^{1}} \leq \Gamma$ we have

$$
|u|_{C^{0}} \leq C_{p}\left\{\left|\operatorname{div}\left(T_{1} \nabla u\right)\right|_{L^{p}}+|u|_{L^{p}}\right\}^{1-e_{p}}
$$

for any $p>1$, where $C_{p}$ depends only on $\Gamma$, Volume $\left(M^{\varepsilon}\right)$ and $p$, and where $e_{p}>0$ with $e_{p} \rightarrow 0$ as $p \rightarrow 1$.

Proof. We prove this using a Sobolev inequality and a standard iteration procedure. In the proof $c_{1}, c_{2} \ldots$ will be constants independent of $\varepsilon$. By a result of Allard and Michael-Simon (see [1], [17]) we know that

$$
\left(\int|v|^{2}\right)^{\frac{1}{2}} \leq c_{1} \int(|\nabla v|+|H||v|)
$$


for $v \in C_{0}^{1}\left(M^{\varepsilon}\right)$, where $\mathrm{H}$ is the mean curvature, $\nabla v$ is the gradient of $\mathrm{v}$ and $c_{1}$ depends only on $\mathrm{n}$. Applying this to $v=u^{s}$ with $u \in C_{0}^{1}\left(M^{\varepsilon}\right)$ and $s=q /(2-q)$ for $1 \leq q<2$, one easily derives the $L^{q}$ Sobolev inequality

$$
\left(\int\left|{ }_{n} v\right|^{2 q /(2-q)}\right)^{(2-q) / 2 q} \leq c_{2}\left(\int\left|\nabla_{n} v\right|^{q}\right)^{1 / q}+\left(\int\left|{ }_{n} v\right|^{q}\right)^{1 / q}
$$

since $|H|$ is bounded on $M^{\varepsilon}$ compact.

Moreover for $u \in C^{2}\left(M^{\varepsilon}\right)$ we have:

$$
-\operatorname{div}\left(T_{1} \nabla|u|\right) \leq\left|\operatorname{div}\left(T_{1} \nabla u\right)\right|
$$

to prove (4.2), let $x_{1}, x_{2}$ be a system of local orthonormal coordinates. Then

$$
\begin{aligned}
\operatorname{div}\left(T_{1} \nabla|u|\right)= & \operatorname{div}\left(T_{1 i j} \frac{\partial u}{\partial x_{i}} \frac{u}{|u|}\right) \\
= & \frac{\partial}{\partial x_{j}}\left(T_{1 i j} \frac{\partial u}{\partial x_{i}}\right) \frac{u}{|u|}+T_{1 i j} \frac{\partial u}{\partial x_{i}} \frac{\partial u}{\partial x_{j}} \frac{1}{|u|} \\
& -T_{1 i j} \frac{\partial u}{\partial x_{i}} \frac{\partial u}{\partial x_{j}} \frac{u^{2}}{|u|^{3}}=\operatorname{div}\left(T_{1} \nabla u\right) \frac{u}{|u|}
\end{aligned}
$$

So we have (4.2).

Now let $k \in \mathbb{R}, k>2$ and integrate by parts to get:

$$
\begin{aligned}
\int|u|^{k-1} \operatorname{div}\left(T_{1} \nabla|u|\right) & =-(k-1) \int|u|^{k-2}(\nabla|u|)\left(T_{1} \nabla|u|\right) \\
& \leq-\left.c_{3}(k-1) \int|u|^{k-2}|\nabla| u\right|^{2}
\end{aligned}
$$

since $T_{1}$ is positive definite. And so by (4.2):

$$
\left.c_{3}(k-1) \int|u|^{k-2}|\nabla| u\right|^{2} \leq \int|u|^{k-1}\left|\operatorname{div}\left(T_{1} \nabla u\right)\right|
$$

(Integration by parts is justified as the integral on the right is finite). Now

$$
\left.\left.\int|\nabla| u\right|^{k / 2}\right|^{2}=\frac{k^{2}}{4} \int|u|^{k-2}|\nabla| u||^{2}
$$

combining this with (4.3) we get

$$
\left.\left.\int|\nabla| u\right|^{k / 2}\right|^{2} \leq\left.\left|c_{4} k \int\right| u\right|^{k-1}\left|\operatorname{div}\left(T_{1} \nabla u\right)\right| \mid .
$$


For $1 \leq q<2$, we apply Hölder's inequality to get

$$
\left.\left.\int|\nabla| u\right|^{k / 2}\right|^{q} \leq c_{5}\left(\left.\left.\int|\nabla| u\right|^{k / 2}\right|^{2}\right)^{q / 2} .
$$

Now apply (4.4) to the right hand sides and (4.1) to the left hand side of the above inequality and we get with $t=q /(2-q)$ :

$$
\begin{aligned}
\left(\int|u|^{k t}\right)^{\frac{1}{t}} & \leq c_{6}\left(\left(\left.\left.\int|\nabla| u\right|^{k / 2}\right|^{q}\right)^{1 / q}+\left(\int|u|^{k q / 2}\right)^{1 / q}\right)^{2} \\
& \leq c_{7}\left(\left(\left.\left.\int|\nabla| u\right|^{k / 2}\right|^{2}\right)^{1 / 2}+\left(\int|u|^{k}\right)^{1 / 2}\right)^{2} \\
& \leq c_{8}\left(\left.\left.\int|\nabla| u\right|^{k / 2}\right|^{2}+\int|u|^{k}\right) \\
& \leq c_{9}\left(k \int|u|^{k-1}\left|\operatorname{div}\left(T_{1} \nabla u\right)\right|+\int|u|^{k-1}|u|\right) .
\end{aligned}
$$

Let $\delta>0$ be small, and apply Hölder's inequality twice to the right hand side of (4.5):

$$
\begin{aligned}
\left(\int|u|^{k t}\right)^{1 / t} & \leq c_{9}\left(k \int|u|^{k-1}\left|\operatorname{div}\left(T_{1} \nabla u\right)\right|+\int|u|^{k-1}|u|\right) \\
& \leq c_{10}\left(\int|u|^{(k-1) t^{1-\delta}}\right)^{1 / t^{1-\delta}}\left(k\left|\operatorname{div}\left(T_{1} \nabla u\right)\right|_{L^{t_{\delta}}}+|u|_{L^{t_{\delta}}}\right) \\
& \leq c_{11}\left(\int|u|^{k t^{1-\delta}}\right)^{(k-1) / k t^{1-\delta}}\left(k\left|\operatorname{div}\left(T_{1} \nabla u\right)\right|_{L^{t_{\delta}}}+|u|_{L^{t_{\delta}}}\right)
\end{aligned}
$$

where $t_{\delta}$ is the conjugate exponent $t^{1-\delta} /\left(t^{1-\delta}-1\right)$ of $t^{1-\delta}$. Now, letting $k=$ $t^{\delta i}, \mathrm{i}=1,2 \ldots\left(\mathrm{t}\right.$ with q close enough to 2 to have $\left.k=t^{\delta}>2\right)$ and $I_{i}=|u|_{L^{t^{\delta i+1}}}$, the above inequality gives us the recursion formula with $\xi=\xi_{i}=t^{\delta i}$ :

$$
\begin{aligned}
I_{i} & \leq\left(c_{11}\right)^{1 / \xi} I_{i-1}^{\frac{\xi-1}{\xi}}\left(\xi \mid \operatorname{div}\left(\left.T_{1} \nabla u\right|_{L^{t_{\delta}}}+|u|_{L^{t_{\delta}}}\right)^{\frac{1}{\xi}}\right. \\
& \leq\left(c_{11} \xi\right)^{\frac{1}{\xi}} I_{i-1}^{\frac{\xi-1}{\xi}}\left(\mid \operatorname{div}\left(\left.T_{1} \nabla u\right|_{L^{t_{\delta}}}+|u|_{L^{t_{\delta}}}\right)\right)^{\frac{1}{\xi}} .
\end{aligned}
$$

Since $\xi>1$, iterating (4.6) gives us:

$$
I_{i} \leq c_{12} t_{i}\left(\mid \operatorname{div}\left(\left.T_{1} \nabla u\right|_{L^{t_{\delta}}}+|u|_{L^{t_{\delta}}}\right)\right)^{\theta_{i}} I_{0}^{s_{i 1}}
$$


where,

$$
\begin{aligned}
s_{i j} & =\frac{\left(t^{\delta i}-1\right)\left(t^{\delta(i-1)}-1\right) \ldots\left(t^{\delta j}-1\right)}{t^{\delta i} t^{\delta(i-1)} \ldots t^{\delta(j-1)}} \quad(1 \leq j \leq i), \\
& =\frac{1}{t^{\delta i}} \quad(j=i+1), \\
t_{i} & =\prod_{j=2}^{i+1}\left(t^{\delta(j-1)}\right)^{s_{i j}} \\
\theta_{i} & =\sum_{j=2}^{i+1} s_{i j} .
\end{aligned}
$$

The idea is to let $i \rightarrow \infty$ in (4.7) and use the fact that $|u|_{C^{0}}=$ limsupI $_{i}$ as $i \rightarrow \infty$. It is easy that $\log t_{i} \leq \log t^{\delta} \sum_{j=1}^{\infty} j t^{-\delta j}<\infty$ and so $c_{10} t_{i} \leq c_{11}$ (which depends on $\delta$ ). Also, $0<s_{i 1}<1$ and thus $I_{0}^{s_{i 1}} \leq c_{12} \leq \max (1, \Gamma)$. Finally it is not hard to show that $\theta_{i}$ is increasing in $i$ and satisfies $\frac{1}{t^{\delta}} \leq$ $\theta_{i} \leq 1$. Denoting $\theta=\theta_{\delta}=\lim _{i \rightarrow \infty} \theta_{i}$, and letting $i \rightarrow \infty$ in (4.7) we get

$$
|u|_{C^{0}} \leq c_{13}\left(\left|\operatorname{div}\left(T_{1} \nabla u\right)\right|_{L^{t_{\delta}}}+|u|_{\left.\left.L^{t_{\delta}}\right)\right)^{\theta_{\delta}}} .\right.
$$

Letting $p=t_{\delta}=t^{1-\delta} /\left(t^{1-\delta}-1\right)$ and recalling that $t=q / q-2$ the above inequality implies lemma 2 .

\section{Schauder estimates.}

Let $M^{\varepsilon}$ be a $C^{3, \beta}$ surface of $\mathbb{R}^{3}$, with $\partial M^{\varepsilon}$ of class $C^{3, \beta}$ for $0<\varepsilon \leq$ $\varepsilon_{1}, 0<\beta \leq 1$ and $\mathrm{L}$ an elliptic differential operator on $C^{2, \alpha}\left(M^{\varepsilon}\right)$. We assume:

a) There exists a finite collection of $C^{3, \beta}$ parametrizations $U=\left\{\left(\Omega_{j}, \Psi_{j}\right)\right\}$ $\mathrm{j}=1, \ldots \mathrm{J}, \Psi_{j}: \Omega_{j} \longrightarrow M^{\varepsilon}$.

b) For each $x \in \Omega_{j}$ there is a closed ball of radius $\varepsilon, B_{\varepsilon}$ with $x \in B_{\varepsilon} \subset \Omega_{j}$.

c) $\sum_{j}\left|\Psi_{j}\right|_{C^{k}} \leq C_{0} \varepsilon^{1-k}$ and $\left|D \Psi_{j}\right|_{C^{0}} \geq C_{0}^{-1}$ for $k=1,2,3$ and for $0<$ $\alpha \leq \beta, \sum_{j}\left|\Psi_{j}\right|_{C^{k, \alpha}} \leq C_{0} \varepsilon^{1-k-\alpha}$ for some constant $C_{0}>0$ independent of $\varepsilon>0$.

d) The metric on $\Omega_{k}$ satisfies

$$
\begin{aligned}
& \lambda I \leq\left(g_{i j}\right) \leq \lambda^{-1} I \\
& \lambda I \leq\left(g^{i j}\right) \leq \lambda^{-1} I
\end{aligned}
$$

for some constant $\lambda$. 
e) (Straightening out the boundary) If $x_{0} \in \Omega \cap \Psi^{-1}\left(\partial M^{\varepsilon}\right),(\Omega, \Psi) \in U$, there exists a $C^{3, \beta}$ parametrization $\Phi$, straightening out the boundary with $\Phi\left(x_{0}\right)=0$

$$
\begin{aligned}
& \Phi: B_{\varepsilon / 4}\left(x_{0}\right) \cap \Omega \longrightarrow \mathbb{R}_{+}^{2}=\left\{x \in \mathbb{R}^{2} / x_{2} \geq 0\right\} \\
& \Phi\left(B_{\varepsilon / 4}\left(x_{0}\right) \cap \Psi^{-1}\left(\partial M^{\varepsilon}\right)\right) \subset \mathbb{R} \times\{0\} \\
& |\Phi|_{C^{k}} \leq C_{0} \varepsilon^{1-k} \\
& |\Phi|_{C^{k, \alpha}} \leq C_{0} \varepsilon^{1-k-\alpha} \quad \text { for } k=1,2,3 \text { and for } 0<\alpha \leq \beta \\
& |D \Phi|_{C^{0}} \geq C_{0}^{-1}
\end{aligned}
$$

f) If $u \in C^{2, \alpha}\left(M^{\varepsilon}\right)$ and $x=\left(x_{1}, x_{2}\right)$ are coordinates for $(\Omega, \Psi) \in U$ then

$$
L u=a_{i j} u_{x_{i} x_{j}}+b_{i} u_{x_{i}}+c u
$$

in $\Omega$, where the coefficients $a_{i j}, b_{i}, c$ are $C^{0, \alpha}$ and satisfy

$$
\begin{gathered}
\lambda I \leq\left(a_{i j}\right) \leq \lambda^{-1} I \\
\sum_{i j}\left(\left|a_{i j}\right|_{C^{0}}+\varepsilon^{\alpha}\left|a_{i j}\right|_{C^{0, \alpha}}\right) \leq C_{0} \\
\sum_{i}\left(\varepsilon\left|b_{i}\right|_{C^{0}}+\varepsilon^{1+\alpha}\left|b_{i}\right|_{C^{0, \alpha}}\right) \leq C_{0} \\
\left(\varepsilon^{2}|c|_{C^{0}}+\varepsilon^{2+\alpha}|c|_{C^{0, \alpha}}\right) \leq C_{0},
\end{gathered}
$$

the constant $\lambda$ and $C_{0}$ don't depend on $\varepsilon$.

Explosions as in c) and f) require that in the case of $\mathrm{H}$-surfaces we attach the strip $S^{\varepsilon}$, in a neighborhood of $\left(q_{i}\right)_{i=1,2}$ with a cut off function satisfying $\left|D^{k} \phi\right| \leq c \varepsilon^{-k}$ and then $\Psi_{j}$ verify these assumptions.

Clearly $M^{\varepsilon}$ constructed in section 3 satisfies a), b), c), d) since the $C^{3, \beta}$ bounds of $\Psi_{j}(\mathrm{j}=1 \ldots \mathrm{J})$ is independent of $\varepsilon$ (see 3.9) and the width of the strip is $\varepsilon$.

Condition e) is obviously true when $x$ is a boundary point of $S_{1}$ or $S_{2}$ as $\left(S_{i}\right)_{i=1,2}$ don't depend on $\varepsilon$. If $x_{0} \in \partial \Omega_{1}=\partial S^{\varepsilon}$, then e) follows from (3.10) and the definition of $S^{\varepsilon}$, since $\partial S^{\varepsilon}$ is locally a graph over $\left[0, l_{0}\right]$ of a function of the type $x \longrightarrow \varepsilon F(x / \varepsilon)$ where $\mathrm{F}$ is $C^{3, \beta}$ and $C_{0}^{-1} \leq|D F| \leq C_{0}$.

$L u=\operatorname{div} T_{1} \nabla u+2 K H u$ is elliptic since $M^{\varepsilon}$ is strictly locally convex. Since the coefficients of $T_{1}$ depend on the coefficients of the second fundamental form (see section 1), their bounds in $C^{0, \alpha}$ depend on bounds of $|\Psi|_{C^{3, \alpha}}$ and we have f).

Finally, under the above assumptions N.Smale proved. 
Lemma 5.1. Let $\sigma>0$. Then for $u \in C^{2, \alpha}\left(M^{\varepsilon}\right)$ we have the following estimates, for $0<\alpha \leq \beta$

$$
\begin{aligned}
|u|_{C^{2}} & \leq \sigma \varepsilon^{\alpha}|u|_{C^{2, \alpha}}+C_{\sigma} \varepsilon^{-2}|u|_{C^{0}} \\
|u|_{C^{1, \alpha}} & \leq \sigma \varepsilon|u|_{C^{2, \alpha}}+C_{\sigma} \varepsilon^{-1-\alpha}|u|_{C^{0}} \\
|u|_{C^{1}} & \leq \sigma \varepsilon^{1+\alpha}|u|_{C^{2, \alpha}}+C_{\sigma} \varepsilon^{-1}|u|_{C^{0}}
\end{aligned}
$$

where $C_{\sigma}$ is a constant depending on $\sigma$ and $C_{0}$.

Lemma 5.2. If $u \in C_{0}^{2, \alpha}\left(M^{\varepsilon}\right)$ and $L u=F, 0<\alpha \leq \beta$ then

$$
|u|_{C^{2, \alpha}} \leq C_{\alpha}\left\{\varepsilon^{-\alpha}|F|_{C^{0}}+|F|_{(\alpha)}+\varepsilon^{-2-\alpha}|u|_{C^{0}}\right\}
$$

for all $\varepsilon<\varepsilon(\alpha)$, where $C_{\alpha}$ depends on $\alpha, C_{0}$, and $\lambda$.

Even if the construction of $M^{\varepsilon}$ satisfies better bounds than assumptions a), c), d), e), f) we can't have an explosion smaller than in Lemma 5.2. This explosion comes from b) and a necessary cut off function on $B_{\varepsilon}$ which verify $\left|D^{k} \phi\right| \leq c \varepsilon^{-k}$ to prove the local Schauder estimate (see [20]).

Since we want to apply, the Schauder fixed point theorem in the Banach space $C_{0}^{3, \alpha}\left(M^{\varepsilon}\right)$, we establish two Lemmas of the same character.

A direct application of local interpolation inequalities (see [8]) (As in lemma 5.1) gives us:

Lemma 5.3. Let $\sigma>0$. Then for $u \in C^{3, \alpha}\left(M^{\varepsilon}\right)$ we have the following estimates, for $0<\alpha \leq \beta$

$$
\begin{aligned}
|u|_{C^{3}} & \leq \sigma \varepsilon^{\alpha}|u|_{C^{3, \alpha}}+C_{\sigma} \varepsilon^{-3}|u|_{C^{0}} \\
|u|_{C^{2, \alpha}} & \leq \sigma \varepsilon|u|_{C^{3, \alpha}}+C_{\sigma} \varepsilon^{-2-\alpha}|u|_{C^{0}} \\
|u|_{C^{2}} & \leq \sigma \varepsilon^{1+\alpha}|u|_{C^{3, \alpha}}+C_{\sigma} \varepsilon^{-2}|u|_{C^{0}} \\
|u|_{C^{1, \alpha}} & \leq \sigma \varepsilon^{2}|u|_{C^{3, \alpha}}+C_{\sigma} \varepsilon^{-1-\alpha}|u|_{C^{0}} \\
|u|_{C^{1}} & \leq \sigma \varepsilon^{2+\alpha}|u|_{C^{3, \alpha}}+C_{\sigma} \varepsilon^{-1}|u|_{C^{0}} \\
|u|_{C^{0, \alpha}} & \leq \sigma \varepsilon^{3}|u|_{C^{3, \alpha}}+C_{\sigma} \varepsilon^{-\alpha}|u|_{C^{0}}
\end{aligned}
$$

where $C_{\sigma}$ is a constant depending on $\sigma$ and $C_{0}$.

In our case $L u=\operatorname{div} T_{1} \nabla u+2 K H u$, we have $a_{i j}=T_{1 i j}, b_{i}=\partial_{j} T_{1 i j}, c=$ $2 K H$ and $M^{\varepsilon}$ of class $C^{4, \beta}$ implies: 
(f') $a_{i j}, b_{i}, c$ are $C^{1, \alpha}$ and satisfy:

$$
\begin{gathered}
\sum_{i j}\left(\left|a_{i j}\right|_{C^{1}}+\left|a_{i j}\right|_{C^{1, \alpha}}\right) \leq C_{0} \\
\sum_{i}\left(\left|b_{i}\right|_{C^{1}}+\left|b_{i}\right|_{C^{1, \alpha}}\right) \leq C_{0} \\
\left(|c|_{C^{1}}+|c|_{C^{1, \alpha}}\right) \leq C_{0}
\end{gathered}
$$

Which gives us:

Lemma 5.4. If $u \in C_{0}^{3, \alpha}\left(M^{\varepsilon}\right)$ and $L u=F, 0<\alpha \leq \beta$ then

$$
|u|_{C^{3, \alpha}} \leq C_{\alpha}\left\{\varepsilon^{-\alpha}|F|_{C^{1}}+|\nabla F|_{(\alpha)}+\varepsilon^{-3-\alpha}|u|_{C^{0}}\right\}
$$

for all $\varepsilon<\varepsilon(\alpha)$, where $C_{\alpha}$ depends on $\alpha, C_{0}$, and $\lambda$

Proof. We have by differentiating the equation $L u=F$

$$
L(\nabla u)=\nabla F-\left(\nabla a_{i j}\right) u_{x_{i} x_{j}}-\left(\nabla b_{i}\right) u_{x_{i}}-(\nabla c) u
$$

By applying lemma 5.2, we have:

$$
\begin{aligned}
|\nabla u|_{C^{2, \alpha}} \leq & C_{\alpha}\left\{\varepsilon^{-\alpha}\left(|F|_{C^{1}}+\left|a_{i j}\right|_{C^{1}}|u|_{C^{2}}+\left|b_{i}\right|_{C^{1}}|u|_{C^{1}}+|c|_{C^{1}}|u|_{C^{0}}\right)\right. \\
& +|\nabla F|_{(\alpha)}+\left|\nabla a_{i j}\right|_{(\alpha)}|u|_{C^{2}}+\left|\nabla b_{i}\right|_{(\alpha)}|u|_{C^{1}}+|\nabla c|_{(\alpha)}|u|_{C^{0}} \\
& +\left|a_{i j}\right|_{C^{1}}\left|D^{2} u\right|_{(\alpha)}+\left|b_{i}\right|_{C^{1}}|\nabla u|_{(\alpha)}+|c|_{C^{1}}|u|_{(\alpha)} \\
& \left.+\varepsilon^{-2-\alpha}|\nabla u|_{C^{0}}\right\}
\end{aligned}
$$

and by (f')):

$$
\begin{aligned}
|\nabla u|_{C^{2, \alpha}} \leq & C_{\alpha}\left\{\varepsilon^{-\alpha}|F|_{C^{1}}+|\nabla F|_{(\alpha)}\right\} \\
& +C_{0} C_{\alpha}\left(\varepsilon^{-\alpha}|u|_{C^{2}}+\left|D^{2} u\right|_{(\alpha)}\right) \\
& +C_{0} C_{\alpha}\left(\varepsilon^{-\alpha}|u|_{C^{1}}+|\nabla u|_{(\alpha)}\right) \\
& +C_{0} C_{\alpha}\left(\varepsilon^{-\alpha}|u|_{C^{0}}+|u|_{(\alpha)}\right) \\
& +C_{\alpha} \varepsilon^{-2-\alpha}|u|_{C^{1}}
\end{aligned}
$$

and by lemma 5.3 we have:

$$
\begin{aligned}
& \varepsilon^{-\alpha}|u|_{C^{2}}+\left|D^{2} u\right|_{(\alpha)} \leq \sigma \varepsilon|u|_{C^{3, \alpha}}+C_{\sigma} \varepsilon^{-2-\alpha}|u|_{C^{0}} \\
& \varepsilon^{-\alpha}|u|_{C^{1}}+|\nabla u|_{(\alpha)} \leq \sigma \varepsilon^{2}|u|_{C^{3, \alpha}}+C_{\sigma} \varepsilon^{-1-\alpha}|u|_{C^{0}} \\
& \varepsilon^{-\alpha}|u|_{C^{0}}+|u|_{(\alpha)} \leq \sigma \varepsilon^{3}|u|_{C^{3, \alpha}}+C_{\sigma^{\prime}} \varepsilon^{-\alpha}|u|_{C^{0}} \\
& \varepsilon^{-2-\alpha}|u|_{C^{1}} \leq \sigma|u|_{C^{3, \alpha}}+C_{\sigma} \varepsilon^{-3-\alpha}|u|_{C^{0}}
\end{aligned}
$$


Then with $\sigma=1 / 2 C_{0} C_{\alpha}$, by substracting the $|u|_{C^{3, \alpha}}$ terms on the right, the lemma is proved.

\section{Eigenvalue bounds and $L^{2}$ estimates.}

Lemma 6.1 is proved as in [20] and we redo here for a complete proof of the theorem.

Lemma 6.1. There exists positive constants $\varepsilon_{1}$ and $d_{0}$ such that for all $\varepsilon<\varepsilon_{1}$

$$
\operatorname{dist}(\operatorname{spec}(L), 0) \geq d_{0}
$$

$\varepsilon_{1}$ and $d_{0}$ depend only on $\max _{M^{\varepsilon}}|2 K H|$ (i.e. on $C_{0}$ ) and $\min _{i=1,2}\left(d_{i}\right), d_{i}=$ $\operatorname{dist}\left(\operatorname{spec}\left(L^{S_{i}}\right), 0\right)$.

Proof. If lemma 6.1 were false, then there would exist a sequence of eigenvalues $\lambda_{k}=\lambda_{k}\left(e_{k}\right) k=1,2 \ldots$ such that $\lambda_{k} \rightarrow 0$ as $k \rightarrow \infty$ (and $\varepsilon_{k} \rightarrow 0$ ), and a sequence of eigenfunctions $u_{k}$ such that

$$
L u_{k}=-\lambda_{k} u_{k} \text { and } \int\left|u_{k}\right|^{2}=1 \text {. }
$$

$c_{1}, c_{2} \ldots$ will be constants depending only on $C_{0}, d_{1}$ and $d_{2}$. First we show that $\left|u_{k}\right|_{C^{0}}$ is bounded. This follows easily from lemma 4.1:

$$
\left|u_{k}\right|_{C^{0}} \leq C_{p}\left\{\left|-\lambda_{k} u_{k}-2 K H u_{k}\right|_{L^{p}}+\left|u_{k}\right|_{L^{p}}\right\}^{1-e_{p}} \text { for } p>1
$$

(Note that lemma 4.1 applies here as $\left|u_{k}\right|_{L^{1}} \leq c_{1}$ ). Now

$$
\left|-\lambda_{k} u_{k}-2 K H u_{k}\right|_{L^{p}} \leq\left(\left|\lambda_{k}\right|+|2 K H|_{C^{0}}\right)\left|u_{k}\right|_{L^{p}},
$$

so $\left|u_{k}\right|_{C^{0}} \leq c_{2}\left|u_{k}\right|_{L^{p}}^{1-e_{p}}$ and $\left|u_{k}\right|_{L^{2}}=1$ imply $\left|u_{k}\right|_{C^{0}} \leq c_{2}$.

Also integrating the eigenvalues equation by parts, we have:

$$
\begin{gathered}
\int u_{k} \operatorname{div} T_{1} \nabla u_{k}+\int 2 K H u_{k}^{2}=-\lambda_{k} \int\left|u_{k}\right|^{2} \\
c_{0} \int\left|\nabla u_{k}\right|^{2} \leq\left(\left|\lambda_{k}\right|+|2 K H|_{C^{0}}\right) \int\left|u_{k}\right|^{2} \\
\left|\nabla u_{k}\right|_{L^{2}}^{2} \leq c_{3}\left|u_{k}\right|_{L^{2}}^{2} \leq c_{3} .
\end{gathered}
$$

Let $\left(B_{5 C_{0} \varepsilon}\left(q_{i}\right)\right)_{i=1,2}$ be small geodesic ball about $q_{i}$ of radius $5 C_{0} \varepsilon$. Since $C_{0}^{-1} \leq\left|D \Psi_{j}\right| \leq C_{0}, B_{2 C_{0} \varepsilon}\left(q_{i}\right)$ contains deformations of the boundary where the strip is attached (see section 3). Let $\bar{S}^{\varepsilon}=\Psi_{1}\left(S^{\varepsilon}\right) \cup B_{5 C_{0} \varepsilon}\left(q_{1}\right) \cup B_{5 C_{0} \varepsilon}\left(q_{2}\right)$ 
Since $\operatorname{Vol}\left(\bar{S}^{\varepsilon}\right) \leq c_{4} \varepsilon$ and $\int\left|u_{k}\right|^{2}=1$, we can assume that

$$
1 / 4 \leq \int_{S_{1} / B_{5 C_{0} \varepsilon}}\left|u_{k}\right|^{2}
$$

(by chosing a subsequence if necessary). Let $\Phi$ be a cut off function,defined on $S_{1}$ by

$$
\Phi(x)= \begin{cases}0 & r<\xi \\ \frac{\log (r / \xi)}{\log \left(1 / \xi^{1 / 2}\right)} & \xi \leq r \leq \xi^{1 / 2} \\ 1 & \xi^{1 / 2}<r\end{cases}
$$

where $\xi^{1 / 2}=3 C_{0} \varepsilon$ and $r(x)=$ geodesic distance from $q_{1}$ to $x$. So $\Phi$ is lipschitz and thus $H^{1}$. By integrating in geodesic polar coordinates

$$
\begin{gathered}
\int_{B_{\xi^{1 / 2}}}|\nabla \Phi|^{2} \sqrt{g} d x \leq \frac{c}{\log \left(\xi^{-1 / 2}\right)^{2}} \int_{r=\xi}^{\xi^{1 / 2}} \frac{d r}{r} \leq \frac{c}{\log \left(\xi^{-1 / 2}\right)} \\
|\nabla \Phi|_{L^{2}} \leq c\left(\log \xi^{-1 / 2}\right)^{-1 / 2} \rightarrow 0 \text { as } \xi, \varepsilon \rightarrow 0 .
\end{gathered}
$$

(Here $\sqrt{g} d x$ is the volume measure in linear coordinates on the geodesic ball $B_{\rho} ; \sqrt{g} d x=\sqrt{g} r d r d \theta$ and we used the fact that $\sqrt{g}$ is bounded). Now let $\Phi_{j} \in C^{2}\left(S_{1}\right) j=1,2 \ldots$ so that $\Phi_{j} \rightarrow \Phi$ in $C^{0}$ and $H^{1}$ norms as $j \rightarrow \infty$ and $\Phi_{j} \equiv 0$ on $B_{2 C_{0} \varepsilon}\left(q_{1}\right)$. Also let $\lambda_{k}$ and $u_{k}$ be as above. Then $v_{k, j}=\Phi_{j} u_{k} \in$ $H_{0}^{1}\left(S_{1}\right)$ and

$$
\begin{aligned}
& \left|v_{k, j}\right|_{C^{0}} \leq c_{4} \\
& \left|v_{k, j}\right|_{L^{2}} \geq 1 / 4 .
\end{aligned}
$$

We have the orthogonal decomposition under $\mathrm{L}$

$$
L^{2}\left(S_{1}\right)=H_{+} \oplus H_{-}
$$

where $H_{+}\left(H_{-}\right)$is the direct sum of the eigenspaces of $L^{S_{1}}$ with positive (negative) eigenvalues. For $w \in L^{2}\left(S_{1}\right)$, let $w^{+}, w^{-}$, be the orthogonal projections onto $H_{+}, H_{-}$respectively. Now

$$
\int\left|v_{k, j}\right|^{2}=\int\left|v_{k, j}^{+}\right|^{2}+\int\left|v_{k, j}^{-}\right|^{2} \leq c_{1}
$$

and so $\left|v_{k, j}^{-}\right|_{C^{0}} \leq c_{5}$ since $H_{-}$is finite dimensional. Thus $\left|v_{k, j}^{+}\right|_{C^{0}} \leq c_{6}$ since $\left|v_{k, j}\right|_{C^{0}} \leq c_{4}$ and $v_{k, j}=v_{k, j}^{+}+v_{k, j}^{-}$. Letting $c_{7}^{-1}=\min _{i=1,2}\left(d_{i}\right)$ we have by standard Hilbert theory:

$$
1 / 4 \leq \int\left|v_{k, j}^{+}\right|^{2}+\int\left|v_{k, j}^{-}\right|^{2} \leq c_{7}\left(\left|\int v_{k, j}^{+} L v_{k, j}\right|+\left|\int v_{k, j}^{-} L v_{k, j}\right|\right)
$$


We show that the terms on the right goes to zero, getting a contradiction when $j \rightarrow \infty$ and $k \rightarrow \infty$. Now

$$
\begin{aligned}
\left|\int v_{k, j}^{-} L v_{k, j}\right| & \leq\left|\int v_{k, j}^{-}\left(-\lambda_{k} v_{k, j}+2 T_{1} \nabla u_{k} \nabla \Phi_{j}+u_{k} \operatorname{div} T_{1} \nabla \Phi_{j}\right)\right| \\
& \leq c_{8}\left(\lambda_{k}+\left|\nabla u_{k}\right|_{L^{2}}\left|\nabla \Phi_{j}\right|_{L^{2}}\left|v_{k, j}^{-}\right|_{C^{0}}+\left|\int v_{k, j}^{-} u_{k} \operatorname{div} T_{1} \nabla \Phi_{j}\right|\right) \\
& \leq c_{9}\left(\lambda_{k}+\left|\nabla u_{k}\right|_{L^{2}}\left|\nabla \Phi_{j}\right|_{L^{2}}+\left|\int v_{k, j}^{-} u_{k} \operatorname{div} T_{1} \nabla \Phi_{j}\right|\right)
\end{aligned}
$$

If we let $j \rightarrow \infty$ and $k \rightarrow \infty$, the first two terms tend to zero since $\left|\nabla u_{k}\right|_{L^{2}}$ is bounded and $\left|\nabla \Phi_{j}\right|_{L^{2}} \rightarrow|\nabla \Phi|_{L^{2}}$ as $j \rightarrow \infty$ and $|\nabla \Phi|_{L^{2}} \rightarrow 0$ as $k \rightarrow \infty$ $(\varepsilon \rightarrow 0)$.

So we must show that the third term on right tends to zero.

Integrating by parts

$$
\begin{aligned}
\left|\int v_{k, j}^{-} u_{k} \operatorname{div} T_{1} \nabla \Phi_{j}\right| & \leq\left|\int \nabla v_{k, j}^{-} u_{k} T_{1} \nabla \Phi_{j}\right|+\left|\int v_{k, j}^{-} \nabla u_{k} T_{1} \nabla \Phi_{j}\right| \\
& \leq c_{10}\left(\left|\nabla v_{k, j}^{-}\right|_{L^{2}}\left|\nabla \Phi_{j}\right|_{L^{2}}+\left|\nabla u_{k}\right|_{L^{2}}\left|\nabla \Phi_{j}\right|_{L^{2}}\right)
\end{aligned}
$$

as remarked above, the second term on the right tends to zero. Since $\left|\nabla \Phi_{j}\right|_{L^{2}} \rightarrow 0$, it suffices to show that $\left|\nabla v_{k, j}^{-}\right|_{L^{2}}$ is bounded. However, this follows from the fact that $H_{-}$is finite dimensional and that $\left|\nabla v_{k, j}^{-}\right|_{L^{2}}$ is bounded. For example $v_{k, j}^{-}=a_{1} \Lambda_{1}+\ldots+a_{I} \Lambda_{I}, I=$ index of $S_{1},\left\{\Lambda_{i}\right\}$ $i=1, \ldots I$ a smooth orthonormal basis for $H_{-}$and $\left(\sum a_{i}^{2}\right)^{1 / 2} \leq c$. Then $\nabla v_{k, j}^{-}=a_{1} \nabla \Lambda_{1} \ldots a_{I} \nabla \Lambda_{I}$ is clearly bounded in $L^{2}$.

Thus we've shown that $\int v_{k, j}^{-} L v_{k, j}$ tends to zero. The proof that $\int v_{k, j}^{+} L v_{k, j}$ tends to zero is identical, except that $\left|\nabla v_{k, j}^{+}\right|_{L^{2}}$ is bounded since $\left|\nabla v_{k, j}^{-}\right|_{L^{2}}$ and $\left|\nabla v_{k, j}\right|_{L^{2}}$ are bounded (independent of $\varepsilon$ ). This completes the proof of lemma 6.1.

For the rest of the paper $H_{+}, H_{-}$will denote the direct sum of the eigenspaces of $\mathrm{L}$ in $L^{2}\left(M^{\varepsilon}\right)$ corresponding to the positive(negative) eigenvalues. So $L^{2}\left(M^{\varepsilon}\right)=H_{+} \oplus H_{-}$is an orthogonal sum, invariant under L. As above, if $u \in L^{2}\left(M^{\varepsilon}\right), u=u^{+}+u^{-}$for unique $u^{+} \in H_{+}, u^{-} \in H_{-}$. We have the following:

Corollary 6.1. For $u \in C_{0}^{3, \alpha}\left(M^{\varepsilon}\right)$ we have

$$
\begin{aligned}
|u|_{L^{2}}^{2} & \leq C_{1}\left(\left|u^{+}\right|_{C^{0}}+\left|u^{-}\right|_{C^{0}}\right)|L u|_{L^{1}} \\
|u|_{H^{1}} & \leq C_{2}|L u|_{L^{2}}
\end{aligned}
$$


where $C_{1}, C_{2}$ depends only on $d_{0}$ and $c_{0}$ the constant of ellipticity of $L u$.

Proof. By standard Hilbert space theory

$$
\int|u|^{2}=\int\left|u^{+}\right|^{2}+\int\left|u^{-}\right|^{2} \leq \frac{1}{d_{0}}\left(\left|\int u^{+} L u\right|+\left|\int u^{-} L u\right|\right)
$$

from which a) follows. Thus

$$
|u|_{L^{2}}^{2} \leq \frac{1}{d_{0}}\left(\left|u^{+} L u\right|_{L^{1}}+\left|u^{-} L u\right|_{L^{1}}\right) \leq C_{1}|u|_{L^{2}}|L u|_{L^{2}}
$$

and so $|u|_{L^{2}} \leq C_{1}|L u|_{L^{2}}$.

Using a) and integrating

$$
u \operatorname{div} T_{1} \nabla u+u 2 K H u=u L u
$$

by parts, we get:

$$
|\nabla u|_{L^{2}}^{2} \leq c_{1}\left(|u|_{L^{2}}^{2}+|u|_{L^{2}}|L u|_{L^{2}}\right) \leq c_{2}|L u|_{L^{2}}^{2}
$$

Which gives us b).

\section{Proof of the theorem 2.1.}

Let $\gamma_{1}, \gamma_{2}, \gamma_{3}$ be the following constants: $\gamma_{1}=2,75, \gamma_{2}=-0,25, \gamma_{3}=$ 1 75. Let $u \in P(\varepsilon, \sigma, \alpha), 0<\varepsilon \leq \varepsilon_{1}, 0<\sigma \leq 1 / 2,0<\alpha \leq \beta$, and recall that $T u=v+w$, where $L v=-(K-1)$ and $L w=-E(u)$. To prove theorem 2.1, we claim that it suffices to establish the following estimates:

$$
\begin{aligned}
|v|_{C^{0}} & \leq K_{1} \varepsilon^{\gamma_{1}-\theta} \\
|v|_{C^{3}, \alpha} & \leq K_{2} \varepsilon^{\gamma_{2}-\alpha-\theta} \\
|v|_{H^{2}} & \leq K_{3} \varepsilon^{\gamma_{3}-\theta} \\
|w|_{C^{0}} & \leq J_{1} \varepsilon^{\beta_{1}-\rho_{1}} \\
|w|_{C^{3, \alpha}} & \leq J_{2} \varepsilon^{\beta_{2}-\rho_{2}} \\
|w|_{H^{2}} & \leq J_{3} \varepsilon^{\beta_{3}-\rho_{3}}
\end{aligned}
$$

for all $\varepsilon<\varepsilon\left(\alpha, C_{0}, d_{0}, S_{1}, S_{2}\right)$ where $K_{1}, K_{2}, K_{3}$ are constants that may depend on $\theta, \alpha, C_{0}, d_{0}, S_{1}, S_{2}$ and where $\theta$ can be taken arbitrarily small; 
$J_{1}, J_{2}, J_{3}$ may depend on $\sigma, \alpha, C_{0}, d_{0}, S_{1}, S_{2} ; \beta_{1}, \beta_{2}, \beta_{3}$ are constants such that

$$
\beta_{1}>2,75, \quad \beta_{2}>-0.25 \quad \beta_{3}>1,75
$$

and finally $\rho_{1}, \rho_{2}, \rho_{3}$ are functions of $\sigma$ and $\alpha$ such that $\rho_{i} \rightarrow 0$ as $\sigma \rightarrow 0$ and $\alpha \rightarrow 0$.

(V1)-(V3) and (W1)-(W3) implies theorem 2.1 for the following reasons: Fix $\sigma=\bar{\sigma}$ and $\alpha=\bar{\alpha}$ so small that $\beta_{i}-\rho_{i}>\gamma_{i} \mathrm{i}=1,2,3$ and then fix $\theta=\bar{\sigma} / 2$. Now of course $K_{i}$ and $J_{i}$ are fixed constants. Letting $\bar{K}=$ $\operatorname{Max}\left\{K_{1}, K_{2}, K_{3}, J_{1}, J_{2}, J_{3}\right\}$, we can take $\bar{\varepsilon}$ so small that:

$$
\begin{aligned}
K_{i} \varepsilon^{\gamma_{i}-\bar{\sigma} / 2}+J_{i} \varepsilon^{\beta_{i}-\rho_{i}} & \leq 2 \bar{K} \varepsilon^{\gamma_{i}-\bar{\sigma} / 2} \leq \varepsilon^{\gamma_{i}-\bar{\sigma}} \quad i \neq 2 \\
K_{2} \varepsilon^{\gamma_{2}-\bar{\sigma} / 2-\bar{\alpha}}+J_{2} \varepsilon^{\beta_{2}-\rho_{2}} & \leq 2 \bar{K} \varepsilon^{\gamma_{2}-\bar{\sigma} / 2-\bar{\alpha}} \leq \varepsilon^{\gamma_{2}-\bar{\sigma}-\bar{\alpha}}
\end{aligned}
$$

for all $\varepsilon<\bar{\varepsilon}$. That is, the right hand inequalities are true for $\varepsilon<\bar{\varepsilon}=$ $(2 \bar{K})^{-2 / \bar{\sigma}}$. Clearly then, by the definition of $P(\varepsilon, \sigma, \alpha), v+w \in P(\varepsilon, \bar{\sigma}, \bar{\alpha})$, for all $\varepsilon<\bar{\varepsilon}$,since the left hand sides of the above inequalities are just upper bounds for $v+w$ in the norms defining $P(\varepsilon, \bar{\sigma}, \bar{\alpha})$. So it suffices to establish (V1)-(V3) and (W1)-(W3).

\section{Estimations of (V1)-(V3).}

Again $c_{1}, c_{2}, \ldots$ will be constants independent of $\varepsilon$. We first establish a $L^{2}$ estimate of $v$ :

From corollary 6.1-b and (3.13)

$$
|v|_{H^{1}} \leq c_{1}|K-1|_{L^{2}} \leq c_{2} \varepsilon^{2,5}
$$

and so by lemma 4.1, applying to $v^{+}$and $v^{-}$, gives us

$$
\left|v^{ \pm}\right|_{C^{0}} \leq C_{p}\left\{\left|\operatorname{div}\left(T_{1} \nabla v^{ \pm}\right)\right|_{L^{p}}+\left|v^{ \pm}\right|_{L^{p}}\right\}^{1-e_{p}} \text { for } p>1
$$

but for $1<p \leq 2$

$$
\begin{aligned}
\left|\operatorname{div}\left(T_{1} \nabla v^{ \pm}\right)\right|_{L^{p}}+\left|v^{ \pm}\right|_{L^{p}} & \leq c_{3}\left(\left|(K-1)^{ \pm}\right|_{L^{p}}+\left|v^{ \pm}\right|_{L^{p}}\right) \\
& \leq c_{4}\left(\left|(K-1)^{ \pm}\right|_{L^{2}}+\left|v^{ \pm}\right|_{L^{2}}\right) \leq c_{5} \varepsilon^{2,5}
\end{aligned}
$$

since $M^{\varepsilon}$ is compact and bounds on $2 \mathrm{KH}$ depend only on $C^{2}$ bounds of parametrizations $\left(|v|_{L^{2}} \leq c_{2} \varepsilon^{2,5}\right.$ due to (7.1)). Thus $\left|v^{ \pm}\right|_{C^{0}} \leq c_{6} \varepsilon^{2,5\left(1-e_{p}\right)}$ and $c_{6}=c_{6}(p)$ (recall $e_{p} \rightarrow 0$ as $p \rightarrow 1$ ).

Combining this, corollary 6.1 -a gives us

$$
|v|_{L^{2}}^{2} \leq c_{7} \varepsilon^{2,5\left(1-e_{p}\right)}|K-1|_{L^{1}} \leq c_{8} \varepsilon^{5,5-2,5 e_{p}}
$$


and

$$
|v|_{L^{2}} \leq c_{9} \varepsilon^{2,75-\theta} \text { with } \theta \text { close to } 0 \text { as } p \rightarrow 1 .
$$

Now we prove (V.1) using lemma 4.1 and (7.2).

$$
|v|_{C^{0}} \leq C_{p}\left\{\left|\operatorname{div}\left(T_{1} \nabla v\right)\right|_{L^{p}}+|v|_{L^{p}}\right\}^{1-e_{p}} \leq c_{10}\left\{|K-1|_{L^{p}}+|v|_{L^{2}}\right\}^{1-e_{p}}
$$

For $p$ close to $1,|K-1|_{L^{p}} \leq c_{11} \varepsilon^{x}$ with $x$ close to 3 (see 3.12 ).

Then when $p \rightarrow 1,|K-1|_{L^{p}} \leq c_{11} \varepsilon^{2,75-\theta}$ and by (7.2):

$$
|v|_{C^{0}} \leq c_{12} \varepsilon^{(2,75-\theta)\left(1-e_{p}\right)}
$$

which gives (V1) for $p$ close to 1 :

$$
|v|_{C^{0}} \leq K_{1} \varepsilon^{2,75-\theta}
$$

(V2) follows from lemma 5.4, (V1) and (3.14):

$$
|v|_{C^{3, \alpha}} \leq C_{\alpha}\left\{\varepsilon^{-\alpha}|K-1|_{C^{1}}+|\nabla(K-1)|_{(\alpha)}+\varepsilon^{-3-\alpha}|v|_{C^{0}}\right\}
$$

then

$$
|v|_{C^{3, \alpha}} \leq K_{2} \varepsilon^{-0,25-\alpha-\theta}
$$

Now lemma 5.3, combined with (V1) and (V2) imply that

$$
\begin{aligned}
|\nabla v|_{C^{0}} & \leq c_{13} \varepsilon^{1,75-\mu_{1}} \\
\left|D^{2} v\right|_{C^{0}} & \leq c_{14} \varepsilon^{0,75-\mu_{1}}
\end{aligned}
$$

where, $c_{13}=c_{13}\left(\mu_{1}\right), c_{14}=c_{14}\left(\mu_{1}\right)$ and $\mu_{1}>0$ can be chosen arbitrarily small.

Now we prove (V3). First of all, by standard elliptic theory (see [8], [18]), there exists a constant $c_{15}$, depending only on $S_{1}$ and $S_{2}$ such that

$$
|Z|_{H^{2}} \leq c_{15}\left\{|L Z|_{L^{2}}+|Z|_{L^{2}}\right\}
$$

for all $Z \in H^{2}\left(S_{i}\right) \cap H_{0}^{1}\left(S_{i}\right), i=1,2$. Let $\phi$ be a cut off function on $S_{1}$ satisfying

$$
\begin{aligned}
& \phi(x)=1 \text { if } x \text { outside } B_{5 C_{0} \varepsilon}\left(q_{1}\right) \\
& \phi(x)=0 \text { if } x \in B_{3 C_{0} \varepsilon}\left(q_{1}\right)
\end{aligned}
$$

and $|\nabla \phi|_{C^{0}} \leq c_{16} \varepsilon^{-1},\left|D^{2} \phi\right|_{C^{0}} \leq c_{17} \varepsilon^{-2}$. 
Let $B_{i}=B_{5 C_{0} \varepsilon\left(q_{i}\right)}, i=1,2$. We will estimate $\int\left|D^{2} v\right|^{2}$ separately, on the pieces $S_{i} / B_{i}, B_{i} \mathrm{i}=1,2$ and $S^{\varepsilon}$. Applying (7.3) to $Z=\phi v$ and using the fact that support of $\left(\nabla^{k} \phi\right), k=1,2$ is contained in $B_{1}$ with $\operatorname{Vol}\left(B_{1}\right) \leq c \varepsilon^{2}$. We have:

$$
\begin{aligned}
|v|_{H^{2}\left(S_{1} / B_{1}\right) \leq} \leq & c_{15}\left\{\left|\phi(K-1)+2 T_{1} \nabla v \nabla \phi+v \operatorname{div} T_{1} \nabla \phi\right|_{L^{2}}+|\phi v|_{L^{2}}\right\} \\
\leq & c_{18}\left\{\varepsilon^{2,5}+\varepsilon^{-1}|\nabla v|_{C^{0}} \operatorname{Vol}\left(B_{1}\right)^{1 / 2}\right. \\
& \left.+|v|_{C^{0}}\left(\varepsilon^{-1}+\varepsilon^{-2}\right) \operatorname{Vol}\left(B_{1}\right)^{1 / 2}+\varepsilon^{2,75}\right\} \\
\leq & c_{19} \varepsilon^{1,75-\mu_{2}}, \quad c_{19}=c_{19}\left(\mu_{2}\right)
\end{aligned}
$$

One can derive the same estimate for $S_{2} / B_{2}$ and so

$$
\left.|v|_{H^{2}\left(M^{\varepsilon} / \overline{S^{\varepsilon}}\right.}\right) \leq c_{20} \varepsilon^{1,75-\mu_{3}}, \quad c_{20}=c_{20}\left(\mu_{3}\right)
$$

With $\overline{S^{\varepsilon}}=B_{1} \cup B_{2} \cup \Psi_{1}\left(S^{\varepsilon}\right)$.

Now for $i=1,2$,

$$
|v|_{H^{2}\left(B_{i}\right)} \leq c_{21}|v|_{C^{2}}\left(\operatorname{Vol}\left(B_{i}\right)\right)^{1 / 2} \leq c_{21} \varepsilon^{1,75-\mu_{4}}, \quad c_{21}=c_{21}\left(\mu_{4}\right)
$$

So we must estimate

$$
\int_{\Psi_{1}\left(S^{\varepsilon}\right)}\left|D^{2} v\right|^{2}=\int_{S^{\varepsilon}}\left|D^{2} v\right|^{2} \sqrt{g} d t d s
$$

For $\left(\Omega_{1}, \Psi_{1}\right)$ we express $L$ as section $\left.5-f^{\prime}\right)$ and as in the proof of lemma 5.4, by differentiating $L v=-(K-1)$ with respect to t we get on $S^{\varepsilon}$ :

$$
-L\left(v_{t}\right)=K_{t}+\partial_{t} a_{i j} v_{x_{i} x_{j}}+\partial_{t} b_{i} v_{x_{i}}+\partial_{t}(2 K H) v
$$

with the coordinates $x=\left(x_{1}, x_{2}\right)=(t, s)$.

Integrating

$$
v_{t} L v_{t}=v_{t} \operatorname{div} T_{1} \nabla v_{t}+v_{t} 2 K H v_{t}
$$

by parts on $S^{\varepsilon}$ we get:

$$
\int_{S^{\varepsilon}}\left|\nabla v_{t}\right|^{2} \leq c_{22}\left\{\left|\int_{S^{\varepsilon}} v_{t} L v_{t}\right|+\left|\int_{S^{\varepsilon}} v_{t} 2 K H v_{t}\right|+\left|\int_{\partial S^{\varepsilon}} v_{t}\left(T_{1} \nabla v_{t}\right) \cdot e\right|\right\}
$$

where e is the outward unit normal to $\partial S^{\varepsilon}$. But now $v$ is zero on the boundary which is parallel to the t-axis.

Then $v_{t}(t, \varepsilon / 2)=v_{t}(t,-\varepsilon / 2)=0$ for $t \in\left[\varepsilon, l_{0}-\varepsilon\right]$ (see (3.10)). Thus

$$
\int_{\partial S^{\varepsilon}} v_{t}\left(T_{1} \nabla v_{t}\right) \cdot e=\int_{\partial S_{1}^{\varepsilon}} v_{t}\left(T_{1} \nabla v_{t}\right) \cdot e+\int_{\partial S_{2}^{\varepsilon}} v_{t}\left(T_{1} \nabla v_{t}\right) \cdot e
$$


where

$$
\begin{aligned}
& \partial S_{1}^{\varepsilon}=\left\{(t, s) \in \partial S^{\varepsilon} / t=0 \text { and } t=l_{0}\right\} \\
& \partial S_{2}^{\varepsilon}=\left\{(t, s) \in \partial S^{\varepsilon} / 0 \leq t \leq \varepsilon \text { and } l_{0}-\varepsilon \leq t \leq l_{0}\right\}
\end{aligned}
$$

Then $\operatorname{Vol}\left(\partial S_{1}^{\varepsilon}\right) \leq c_{23} \varepsilon$ and $\operatorname{Vol}\left(\partial S_{2}^{\varepsilon}\right) \leq c_{23} \varepsilon$ therefore

$$
\left|\int_{\partial S^{\varepsilon}} v_{t}\left(T_{1} \nabla v_{t}\right) \cdot e\right| \leq c_{24} \varepsilon|\nabla v|_{C^{0}}\left|D^{2} v\right|_{C^{0}} \leq c_{25} \varepsilon^{3,5-\mu_{5}}, \quad c_{25}=c_{25}\left(\mu_{5}\right)
$$

and (7.7) becomes

$$
\begin{aligned}
\int_{S^{\varepsilon}}\left|\nabla v_{t}\right|^{2} & \leq c_{22}\left|\int_{S^{\varepsilon}} v_{t} L v_{t}\right|+c_{26}|\nabla v|_{C^{0}}^{2} \varepsilon+c_{27} \varepsilon^{3,5-\mu_{5}} \\
& \leq c_{22}\left|\int_{S^{\varepsilon}} v_{t} L v_{t}\right|+c_{28} \varepsilon^{3,5-\mu_{5}}, \quad c_{28}=c_{28}\left(\mu_{5}\right) .
\end{aligned}
$$

The first term on the right hand side of (7.8) is estimated by using (7.6) and (V1)-(V3).

$$
\left|\int_{S^{\varepsilon}} v_{t} L v_{t}\right| \leq c_{29}\left\{\int_{S^{\varepsilon}}\left|v_{t}\right|\left|K_{t}\right|+\left|v_{t}\right|\left|\partial_{t} a_{i j} v_{x_{i} x_{j}}\right|+\left|v_{t}\right|\left|\partial_{t} b_{i} v_{x_{i}}\right|+\left|v_{t}\right|\left|\partial_{t} c v\right|\right\} .
$$

Then by (3.14) and by section $\left.5-f^{\prime}\right)$ :

$$
\begin{aligned}
\left|\int_{S^{\varepsilon}} v_{t} L v_{t}\right| & \leq c_{30}\left\{|\nabla v|_{C^{0}}|K-1|_{C^{1}} \varepsilon+|v|_{C^{1}}|v|_{C^{2}} \varepsilon+|v|_{C^{1}}^{2} \varepsilon+|v|_{C^{1}}|v|_{C^{0}} \varepsilon\right\} \\
& \leq c_{31} \varepsilon^{3,5-\mu_{6}}, \quad c_{31}=c_{31}\left(\mu_{6}\right)
\end{aligned}
$$

so together with $(7.8)$

$$
\int_{S^{\varepsilon}}\left|\nabla v_{t}\right|^{2} \leq c_{32} \varepsilon^{3,5-\mu_{7}}, c_{32}=c_{32}\left(\mu_{7}\right) .
$$

Now, we claim that for $x \in S^{\varepsilon}$

$$
\left|D^{2} v\right| \leq c_{33}\left\{\left|\nabla v_{t}\right|+|\nabla v|+|v|+|K-1|\right\}
$$

This follows from the equation $L v=-(K-1)$ and the fact that $\mathrm{L}$ is uniformly elliptic (independent of $\varepsilon$ ).

$L v=-(K-1)$ is of the form $a_{i j} u_{x_{i} x_{j}}+$ lower order terms $=F$. Since $a_{22} \geq \lambda_{0}$, we get

$$
\left|u_{s s}\right| \leq c_{34}\left\{\left|u_{t t}\right|+\left|u_{t s}\right|+\mid \text { lower order terms }|+| F \mid\right\} .
$$


However

$$
\left|u_{t t}\right|+\left|u_{t s}\right| \leq c_{35}\left|\nabla v_{t}\right|
$$

and so (7.10) follows.

Combining (7.9) with (7.10) we get

$$
\int_{S^{\varepsilon}}\left|D^{2} v\right|^{2} \leq c_{36} \varepsilon^{3,5-\mu_{8}}, c_{36}=c_{36}\left(\mu_{8}\right) .
$$

Finally putting together (7.4), (7.5) and (7.11) we get

$$
|v|_{H^{2}} \leq K_{3} \varepsilon^{1,75-\mu_{9}}
$$

where $\mu_{9}$ can be taken arbitrarily small.

\section{Estimation of (W1)-(W3).}

We prove (W1)-(W3) in a manner similar to the proofs of (V1)-(V3) and since $L w=-E(u)$, we will therefore need to estimate $E(u)$ in the $C^{0}, C^{0, \alpha}, C^{1}, C^{1, \alpha}$ and $L^{p}$ norms.To do this we prove the following: 
Lemma 7.1. For $u \in P(\varepsilon, \sigma, \alpha)$ we have:
a) $|E(u)| \leq K_{4}\left(|u|^{2}+|\nabla u|^{2}+\left|D^{2} u\right|^{2}\right)$ (pointwise)
b) $|\nabla E(u)| \leq K_{5}\left(|u|^{2}+|\nabla u|^{2}+\left|D^{2} u\right|^{2}+\left|D^{3} u\right|\left(|u|+|\nabla u|+\left|D^{2} u\right|\right)\right)$ (pointwise)
c) $|E(u)|_{C^{0}} \leq K_{6} \varepsilon^{1,5-2 \sigma}$
d) $|E(u)|_{C^{1}} \leq K_{7} \varepsilon^{0,5-2 \sigma}$
e) $|\nabla E(u)|_{\alpha} \leq K_{8} \varepsilon^{0,5-2 \sigma-\alpha}$

for all $\varepsilon<\varepsilon_{1}, \varepsilon_{1}$ sufficiently small and $K_{4}, \ldots K_{8}$ are constants depending only on $C_{0}, \lambda_{0}$ and $\alpha$.

Proof. First note that c), d) follows from a), b) and the definition of $P(\varepsilon, \sigma, \alpha)$. To prove a), b), e) recall that

$$
E(u)=\int_{0}^{1}(1-\tau) \frac{d^{2} K}{d \tau^{2}}(\tau u) d \tau
$$

So it suffices to show that $\left|\frac{d^{2} K}{d \tau^{2}}(\tau u)\right|,\left|\nabla \frac{d^{2} K}{d \tau^{2}}(\tau u)\right|$ and $\left|\nabla \frac{d^{2} K}{d \tau^{2}}(\tau u)\right|_{(\alpha)}$ are bounded by the quantities on the right hand side of a),b) and e), respectively for $0 \leq \tau \leq 1$.

Let $(\Omega, \Psi) \in U$. Since $\psi(\tau)=\psi+\tau u N$ is a local parametrization of $M_{\tau u}$, we have by (3.7):

$$
K(\tau)=\frac{b_{11}(\tau) b_{22}(\tau)-b_{12}(\tau)^{2}}{\left(g_{11}(\tau) g_{22}(\tau)-g_{12}(\tau)^{2}\right)^{2}}
$$

where we've abbreviated $K(\tau)=K(\tau u)$, with

$$
\begin{aligned}
& g_{i j}(\tau)=\left\langle\psi_{x_{i}}(\tau), \psi_{x_{j}}(\tau)\right\rangle \\
& b_{i j}(\tau)=\left\langle\psi_{x_{1}}(\tau) \wedge \psi_{x_{2}}(\tau), \psi_{x_{i} x_{j}}(\tau)\right\rangle
\end{aligned}
$$

where

$$
\psi_{x_{i}}(\tau)=\psi_{x_{i}}+\tau u N_{x_{i}}+\tau u_{x_{i}} N
$$

Then

$$
\begin{aligned}
\frac{d^{2} K}{d \tau^{2}}(\tau u)= & K(\tau u)^{\prime \prime}=\left(g^{-2}\right)^{\prime \prime} b+2\left(g^{-2}\right)^{\prime} b^{\prime}+\left(g^{-2}\right) b^{\prime \prime} \\
\nabla \frac{d^{2} K}{d \tau^{2}}(\tau u)= & \nabla K(\tau u)^{\prime \prime}=b \nabla\left(g^{-2}\right)^{\prime \prime}+\left(g^{-2}\right)^{\prime \prime} \nabla b \\
& +2 b^{\prime} \nabla\left(g^{-2}\right)^{\prime}+2\left(g^{-2}\right)^{\prime} \nabla b^{\prime}+b^{\prime \prime} \nabla\left(g^{-2}\right)+\left(g^{-2}\right) \nabla b^{\prime \prime}
\end{aligned}
$$


where $g=\operatorname{det}\left(g_{i j}(\tau)\right)$ and $b=\operatorname{det}\left(b_{i j}(\tau)\right)$.

And by appendix 1g)-1i) and 1s)-1u) we obtain a); by appendix 1g)-1l) and $1 \mathrm{~s})-1 \mathrm{x}$ ) we prove $\mathrm{b}$ ) and e).

Proof of (W1)-(W3). In order to prove (W1)-(W3) we will first have to estimate $E(u)$ in $L^{1}, L^{2}$ and $L^{p}, p \geq 1, p$ near of $1 . \quad \nu_{1}, \nu_{2} \ldots$ will denote functions of $\sigma$ and $\alpha$ that tend to zero as $\sigma$ and $\alpha$ tend to zero. $c_{1}, c_{2} \ldots$ will be constants independent of $\varepsilon$.

By lemma 5.3, we know that

$$
|\nabla u|_{C^{0}} \leq c_{1} \varepsilon^{1,75-\sigma}
$$

and

$$
\left|D^{2} u\right|_{C^{0}} \leq c_{1} \varepsilon^{0,75-\sigma} .
$$

First we estimate $|E(u)|_{L^{1}}$.

By lemma 7.1-a) we get by the definition of $P(\varepsilon, \sigma, \alpha)$

$$
\begin{aligned}
|E(u)|_{L^{1}} & \leq K_{4} \int\left(|u|^{2}+|\nabla u|^{2}+\left|D^{2} u\right|^{2}\right) \\
& \leq c_{2}|u|_{H^{2}}^{2} \leq c_{3} \varepsilon^{3,5-2 \sigma}
\end{aligned}
$$

which implies

$$
|E(u)|_{L^{1}} \leq c_{3} \varepsilon^{3,5-\nu_{1}} ;
$$

with $p=1+\delta$ it's easy to see that

$$
|E(u)|_{L^{p}} \leq c_{4} \varepsilon^{3,5-\nu_{2}}
$$

with $c_{4}$ depending on $\delta$.

$$
\begin{aligned}
|E(u)|_{L^{2}} & \leq c_{5}\left(|u|_{L^{4}}^{2}+|\nabla u|_{L^{4}}^{2}+\left|D^{2} u\right|_{L^{4}}^{2}\right) \\
& \leq c_{5}\left(|u|_{C^{0}}|u|_{L^{2}}+|\nabla u|_{C^{0}}|\nabla u|_{L^{2}}+\left|D^{2} u\right|_{C^{0}}\left|D^{2} u\right|_{L^{2}}\right) \\
& \leq c_{6}|u|_{C^{2}}|u|_{H^{2}}
\end{aligned}
$$

which implies that

$$
|E(u)|_{L^{2}} \leq c_{7} \varepsilon^{2,5-\nu_{3}} .
$$

First we prove a $L^{2}$ estimate of $w$.

By corollary 6.1-b):

$$
|w|_{H^{1}} \leq c_{8}|E(u)|_{L^{2}} \leq c_{9} \varepsilon^{2,5-\nu_{3}}
$$


Bridge principle for constant and positive Gauss curvature surfaces 541

applying lemma 4.1 to $w^{ \pm}$we get, after setting $p=1+\delta(\delta$ close to 0$)$ :

$$
\begin{aligned}
\left|w^{ \pm}\right|_{C^{0}} & \leq C_{p}\left\{|E(u)|_{L^{2}}+|w|_{L^{2}}\right\}^{1-e_{p}} \\
& \leq c_{10}\left(\varepsilon^{2,5-\nu_{3}}\right)^{1-e_{p}} \leq c_{11} \varepsilon^{2,5-\nu_{4}}
\end{aligned}
$$

Now we use corollary $6.1-a)$ :

$$
\begin{aligned}
|w|_{L^{2}}^{2} & \leq c_{12} \varepsilon^{2,5-\nu_{4}}|E(u)|_{L^{1}} \\
& \leq c_{13} \varepsilon^{6-\nu_{5}} .
\end{aligned}
$$

Then

$$
|w|_{L^{2}} \leq c_{14} \varepsilon^{3-\nu_{6}}
$$

Now we prove (W1) using lemma 4.1 and (7.13)

$$
|w|_{C^{0}} \leq c_{15}\left\{|E(u)|_{L^{p}}+|w|_{L^{2}}\right\}^{1-e_{p}}
$$

which give (W1) after setting $p=1+\delta$, with $\beta_{1}=3$ and $\rho_{1}=\nu_{7}$.

$$
|w|_{C^{0}} \leq J_{1} \varepsilon^{3-\nu_{7}}
$$

(W2) follows from lemma 5.4 and lemma 7.1:

$$
\begin{aligned}
|w|_{C^{3, \alpha}} & \leq c_{16}\left\{\varepsilon^{-\alpha}|E(u)|_{C^{1}}+|\nabla E(u)|_{(\alpha)}+\varepsilon^{-3-\alpha}|w|_{C^{0}}\right\} \\
& \leq c_{17} \varepsilon^{-\alpha-\nu_{8}}
\end{aligned}
$$

which give (W2) with $\beta_{2}=-\alpha$ and $\rho_{2}=\nu_{8}$.

We prove (W3) like (V3).

Lemma 5.3 implies that

$$
\begin{aligned}
|\nabla w|_{C^{0}} & \leq c_{18} \varepsilon^{2-\nu_{9}} \\
\left|D^{2} w\right|_{C^{0}} & \leq c_{19} \varepsilon^{1-\nu_{10}}
\end{aligned}
$$

As in (V3) it follows by (7.3):

$$
|w|_{H^{2}\left(S_{1} / B_{1}\right)} \leq c_{20}\left\{\left|\phi E(u)+2 T_{1} \nabla w \nabla \phi+w \operatorname{div} T_{1} \nabla \phi\right|_{L^{2}}+|\phi w|_{L^{2}}\right\}
$$

$$
\leq c_{21} \varepsilon^{2-\nu_{11}}
$$

and

$$
\begin{aligned}
|w|_{H^{2}\left(B_{i}\right)} & \leq c_{22}\left|D^{2} w\right|_{C^{0}} \varepsilon \\
& \leq c_{23} \varepsilon^{2-\nu_{12}}
\end{aligned}
$$


Since

$$
|w|_{H^{2}\left(S^{\varepsilon}\right)} \leq c_{24}|w|_{C^{2} \varepsilon^{1 / 2}} \leq c_{25} \varepsilon^{1,5-\nu_{13}},
$$

we must estimate $\int_{\psi_{1}\left(S^{\varepsilon}\right)}\left|D^{2} w\right|^{2}$ and we proceed as in (V3).

By differentiating $L w=-E(u)$ with respect to t we get on $S^{\varepsilon}$ :

$$
-L w_{t}=\partial_{t} E(u)+\partial_{t} a_{i j} w_{x_{i} x_{j}}+\partial_{t} b_{i} w_{x_{i}}+\partial_{t}(2 K H) w .
$$

And integrating by parts on $S^{\varepsilon}, w_{t} L w_{t}$ we get:

$$
\begin{aligned}
\int_{S^{\varepsilon}}\left|\nabla w_{t}\right|^{2} \leq & c_{26}\left\{\left|\int_{S^{\varepsilon}} w_{t} L w_{t}\right|+\left|\int_{S^{\varepsilon}} w_{t} 2 K H w_{t}\right|\right. \\
& \left.+\left|\int_{\partial S^{\varepsilon}} w_{t}\left(T_{1} \nabla w_{t}\right) \cdot e\right|\right\}
\end{aligned}
$$

and as in (V3):

$$
\left|\int_{\partial S^{\varepsilon}} w_{t}\left(T_{1} \nabla w_{t}\right) \cdot e\right| \leq c_{27} \varepsilon|\nabla w|_{C^{0}}\left|D^{2} w\right|_{C^{0}} \leq c_{28} \varepsilon^{4-\nu_{14}}
$$

and

$$
\left|\int_{S^{\varepsilon}} w_{t} 2 K H w_{t}\right| \leq c_{29}|\nabla w|_{C^{0}}^{2} \varepsilon \leq c_{30} \varepsilon^{4-\nu_{15}} .
$$

Moreover by (7.16)

$$
\begin{aligned}
\left|\int_{S^{\varepsilon}} w_{t} L w_{t}\right| \leq & c_{31}\left\{|\nabla w|_{L^{2}}|\nabla E(u)|_{L^{2}}+|\nabla w|_{C^{0}}\left|D^{2} w\right|_{C^{0}} \varepsilon\right. \\
& \left.+|\nabla w|_{C^{0}}^{2} \varepsilon+|\nabla w|_{C^{0}}|w|_{C^{0}} \varepsilon\right\} .
\end{aligned}
$$

By lemma 7.1-b) we get

$$
\begin{aligned}
|\nabla E(u)|_{L^{2}} \leq & c_{32}\left(|u|_{L^{4}}^{2}+|\nabla u|_{L^{4}}^{2}+\left|D^{2} u\right|_{L^{4}}^{2}\right. \\
& \left.\quad+\left|\left(|u|+|\nabla u|+\left|D^{2} u\right|\right)\right| D^{3} u||_{L^{2}}\right) \\
\leq & c_{33}\left(|u|_{C^{2}}|u|_{H^{2}}+\left|D^{3} u\right|_{C^{0}}|u|_{H^{2}}\right) \\
\leq & c_{34}\left(\varepsilon^{0,75-\sigma} \varepsilon^{1,75-\sigma}+\varepsilon^{-0,25-\sigma} \varepsilon^{1,75-\sigma}\right) \leq c_{35} \varepsilon^{1,5-2 \sigma} .
\end{aligned}
$$

Then by (7.12) and the previous estimate:

$$
\begin{aligned}
\left|\int_{S^{\varepsilon}} w_{t} L w_{t}\right| \leq & c_{36}\left(\varepsilon^{2,5-\nu_{3}} \varepsilon^{1,5-\nu_{16}}+\varepsilon^{2-\nu_{9}} \varepsilon^{1-\nu_{10}} \varepsilon+\varepsilon \varepsilon^{4-\nu_{17}}\right. \\
& \left.\quad+\varepsilon \varepsilon^{2-\nu_{9}} \varepsilon^{3-\nu_{7}}\right) \\
\leq & c_{37} \varepsilon^{4-\nu_{18}} .
\end{aligned}
$$


Bridge principle for constant and positive Gauss curvature surfaces 543

Then (7.17), (7.18), (7.19) and (7.20) give

$$
\int_{S^{\varepsilon}}\left|\nabla w_{t}\right|^{2} \leq c_{38} \varepsilon^{4-\nu_{19}}
$$

Since (by (7.10))

$$
\left|D^{2} w\right| \leq c\left\{\left|\nabla w_{t}\right|+|\nabla w|+|w|+|E(u)|\right\}
$$

we get by (7.21), (W1) and lemma 7.1-c):

$$
\begin{aligned}
\int_{S^{\varepsilon}}\left|D^{2} w\right|^{2} & \leq c_{39}\left\{\int_{S^{\varepsilon}}\left|\nabla w_{t}\right|^{2}+|\nabla w|_{C^{0}}^{2} \varepsilon+|w|_{C^{0}}^{2} \varepsilon+|E(u)|_{C^{0}}^{2} \varepsilon\right\} \\
& \leq c_{40} \varepsilon^{4-\nu_{20}}
\end{aligned}
$$

which gives (W3) by (7.14), (7.15) with $\beta_{3}=2$.

\section{Appendix.}

Proposition 1. There exists a constant $K_{9}$ depending only on $C_{0}$ and $\lambda_{0}$ such that the following estimates hold, for $0 \leq \tau \leq 1$, and for $\varepsilon$ sufficiently small:

(a)

$$
\begin{gathered}
\sum_{i, j}\left|g_{i j}\right| \leq K_{9} \\
\sum_{i, j}\left|g_{i j}\right|_{(\alpha)} \leq K_{9} \\
\sum_{i, j}\left|g_{i j}^{\prime}\right| \leq K_{9}\left(|u|+|\nabla u|^{2}\right) \\
\sum_{i, j}\left|g_{i j}^{\prime}\right|_{(\alpha)} \leq K_{9} \varepsilon^{2,75-\sigma-\alpha} \\
\sum_{i, j}\left|g_{i j}^{\prime \prime}\right| \leq K_{9}\left(|u|^{2}+|\nabla u|^{2}\right) \\
\sum_{i, j}\left|g_{i j}^{\prime \prime}\right|_{(\alpha)} \leq K_{9} \varepsilon^{3,5-2 \sigma-\alpha} \\
\sum_{i, j}\left|g_{i j x_{k}}\right| \leq K_{9} \\
\sum_{i, j}\left|g_{i j x_{k}}\right|_{(\alpha)} \leq K_{9}
\end{gathered}
$$

(d) 
(e)

$$
\begin{gathered}
\sum_{i, j}\left|g_{i j x_{k}}^{\prime}\right| \leq K_{9}(|u|+|\nabla u|) \\
\sum_{i, j}\left|g_{i j x_{k}}^{\prime}\right|_{(\alpha)} \leq K_{9} \varepsilon^{1,75-\sigma-\alpha} \\
\sum_{i, j}\left|g_{i j x_{k}}^{\prime \prime}\right| \leq K_{9}\left(|u|^{2}+|u||\nabla u|+\left|D^{2} u\right||\nabla u|\right) \\
\sum_{i, j}\left|g_{i j x_{k}}^{\prime \prime}\right|_{(\alpha)} \leq K_{9} \varepsilon^{2,5-2 \sigma-\alpha}
\end{gathered}
$$

(f)

(g)

$$
\begin{gathered}
\sum_{i, j}|g| \leq K_{9} \\
\sum_{i, j}|g|_{(\alpha)} \leq K_{9} \\
\sum_{i, j}\left|g^{\prime}\right| \leq K_{9}\left(|u|+|\nabla u|^{2}\right) \\
\sum_{i, j}\left|g^{\prime}\right|_{(\alpha)} \leq K_{9} \varepsilon^{2,75-\sigma-\alpha} \\
\sum_{i, j}\left|g^{\prime \prime}\right| \leq K_{9}\left(|u|^{2}+|\nabla u|^{2}\right) \\
\sum_{i, j}\left|g^{\prime \prime}\right|_{(\alpha)} \leq K_{9} \varepsilon^{3,5-2 \sigma-\alpha} \\
\sum_{i, j}\left|g_{x_{k}}^{\prime}\right|_{(\alpha)}^{\prime} \leq K_{9} \varepsilon^{1,75-\sigma-\alpha} \\
\sum_{i, j}\left|g_{x_{k}}\right| \leq K_{9} \\
\sum_{i, j}\left|g_{x_{k}}\right|_{(\alpha)} \leq K_{9}
\end{gathered}
$$

(k) 
Bridge principle for constant and positive Gauss curvature surfaces 545

(l)

$$
\begin{aligned}
\sum_{i, j}\left|g_{x_{k}}^{\prime \prime}\right| & \leq K_{9}\left(|u|^{2}+|\nabla u|^{2}+|\nabla u|\left|D^{2} u\right|\right) \\
\sum_{i, j}\left|g_{x_{k}}^{\prime \prime}\right|_{(\alpha)} & \leq K_{9} \varepsilon^{2,5-2 \sigma-\alpha}
\end{aligned}
$$

(m)

$$
\sum_{i, j}\left|b_{i j}\right| \leq K_{9}
$$

$$
\sum_{i, j}\left|b_{i j}\right|_{(\alpha)} \leq K_{9}
$$

(n)

$$
\sum_{i, j}\left|b_{i j}^{\prime}\right| \leq K_{9}\left(|u|+|\nabla u|+\left|D^{2} u\right|\right)
$$

(o)

$$
\sum_{i, j}\left|b_{i j}^{\prime}\right|_{(\alpha)} \leq K_{9} \varepsilon^{0,75-\sigma-\alpha}
$$

(p)

$$
\begin{aligned}
\sum_{i, j}\left|b_{i j}^{\prime \prime}\right| & \leq K_{9}\left(|u|^{2}+|\nabla u|^{2}+|u|\left|D^{2} u\right|+|\nabla u|\left|D^{2} u\right|\right) \\
\sum_{i, j}\left|b_{i j}^{\prime \prime}\right|_{(\alpha)} & \leq K_{9} \varepsilon^{2,5-2 \sigma-\alpha}
\end{aligned}
$$

(q)

$$
\begin{gathered}
\sum_{i, j}\left|b_{i j x_{k}}\right| \leq K_{9}\left(1+\left|D^{3} u\right|\right) \\
\sum_{i, j}\left|b_{i j x_{k}}\right|_{(\alpha)} \leq K_{9} \varepsilon^{-0,25-\sigma-\alpha} \\
\sum_{i, j}\left|b_{i j x_{k}}^{\prime}\right| \leq K_{9}\left(|u|+|\nabla u|+\left|D^{2} u\right|+\left|D^{3} u\right|\right) \\
\sum_{i, j}\left|b_{i j x_{k}}^{\prime}\right|_{(\alpha)} \leq K_{9} \varepsilon^{-0,25-\sigma-\alpha}
\end{gathered}
$$

(r)

$$
\begin{aligned}
\sum_{i, j}\left|b_{i j x_{k}}^{\prime \prime}\right| & \leq K_{9}\left(|u|^{2}+|\nabla u|^{2}+\left|D^{2} u\right|^{2}+|u|\left|D^{3} u\right|+|\nabla u|\left|D^{3} u\right|\right) \\
\sum_{i, j}\left|b_{i j x_{k}}^{\prime \prime}\right|_{(\alpha)} & \leq K_{9} \varepsilon^{1,5-2 \sigma-\alpha}
\end{aligned}
$$


(s)

$$
\sum_{i, j}|b| \leq K_{9}
$$

$$
\sum_{i, j}|b|_{(\alpha)} \leq K_{9}
$$

(t)

$$
\begin{aligned}
\sum_{i, j}\left|b^{\prime}\right| & \leq K_{9}\left(|u|+|\nabla u|+\left|D^{2} u\right|\right) \\
\sum_{i, j}\left|b^{\prime}\right|_{(\alpha)} & \leq K_{9} \varepsilon^{0,75-\sigma-\alpha}
\end{aligned}
$$

(u)

$$
\begin{aligned}
\sum_{i, j}\left|b^{\prime \prime}\right| & \leq K_{9}\left(|u|^{2}+|\nabla u|^{2}+\left|D^{2} u\right|^{2}\right) \\
\sum_{i, j}\left|b^{\prime \prime}\right|_{(\alpha)} & \leq K_{9} \varepsilon^{1,5-2 \sigma-\alpha}
\end{aligned}
$$

(v)

$$
\sum_{i, j}\left|b_{x_{k}}\right| \leq K_{9}\left(1+\left|D^{3} u\right|\right)
$$

(w)

$$
\sum_{i, j}\left|b_{x_{k}}\right|_{(\alpha)} \leq K_{9} \varepsilon^{-0,25-\sigma-\alpha}
$$

$$
\sum_{i, j}\left|b_{x_{k}}^{\prime}\right| \leq K_{9}\left(|u|+|\nabla u|+\left|D^{2} u\right|+\left|D^{3} u\right|\right)
$$

$$
\sum_{i, j}\left|b_{x_{k}}^{\prime}\right|_{(\alpha)} \leq K_{9} \varepsilon^{-0,25-\sigma-\alpha}
$$

$$
\sum_{i, j}\left|b_{x_{k}}^{\prime \prime}\right| \leq K_{9}\left(|u|^{2}+|\nabla u|^{2}+\left|D^{2} u\right|^{2}+|u|\left|D^{3} u\right|\right.
$$

(x)

$$
\left.+|\nabla u|\left|D^{3} u\right|+\left|D^{2} u\right|\left|D^{3} u\right|\right)
$$

$$
\sum_{i, j}\left|b_{x_{k}}^{\prime \prime}\right|_{(\alpha)} \leq K_{9} \varepsilon^{0,5-2 \sigma-\alpha}
$$


Bridge principle for constant and positive Gauss curvature surfaces 547

Proof of the proposition 1.

As usual $c_{1}, c_{2} \ldots$ will be constants depending only on $C_{0}$ and $\lambda_{0}$. Since $u \in P(\varepsilon, \sigma, \alpha)$, it follows from lemma 5.3 that:

$$
\begin{aligned}
|u|_{C^{3}} & \leq c_{1} \varepsilon^{-0,25-\sigma} \\
|u|_{C^{2, \alpha}} & \leq c_{1} \varepsilon^{0,75-\sigma-\alpha} \\
|u|_{C^{2}} & \leq c_{1} \varepsilon^{0,75-\sigma} \\
|u|_{C^{1, \alpha}} & \leq c_{1} \varepsilon^{1,75-\sigma-\alpha} \\
|u|_{C^{1}} & \leq c_{1} \varepsilon^{1,75-\sigma} \\
|u|_{C^{0, \alpha}} & \leq c_{1} \varepsilon^{2,75-\sigma-\alpha}
\end{aligned}
$$

Moreover we get from the expression of $\psi_{x_{i}}(\tau)$ (see lemma 7.1):

$$
\begin{aligned}
g_{i j}(\tau)= & \left\langle\psi_{x_{i}}, \psi_{x_{j}}\right\rangle+\tau u\left(\left\langle\psi_{x_{i}}, N_{x_{j}}\right\rangle+\left\langle N_{x_{i}}, \psi_{x_{j}}\right\rangle\right) \\
& +\tau^{2} u_{x_{i}} u_{x_{j}}+\tau^{2} u^{2}\left\langle N_{x_{i}}, N_{x_{j}}\right\rangle
\end{aligned}
$$

Since $N_{x_{i}} \in T M$.

Using the fact that $|u| \leq 1,|\nabla u| \leq 1$ and $\left|D^{2} u\right| \leq 1$ so that higher powers of these are dominated by lower powers, using $|\Psi|_{C^{4, \beta}}$ is bounded (independent of $\varepsilon$ ) it is straightforward to establish 1a)-1f) by derivation.

By example:

$$
\begin{aligned}
g_{i j x_{k}}^{\prime}= & u_{x_{k}}\left(\left\langle\psi_{x_{i}}, N_{x_{j}}\right\rangle+\left\langle N_{x_{i}}, \psi_{x_{j}}\right\rangle\right)+u\left(\left\langle\psi_{x_{i}}, N_{x_{j}}\right\rangle+\left\langle N_{x_{i}}, \psi_{x_{j}}\right\rangle\right)_{x_{k}} \\
& +2 \tau u_{x_{i} x_{k}} u_{x_{j}}+2 \tau u_{x_{i}} u_{x_{j} x_{k}}+4 \tau u_{x_{k}} u\left\langle N_{x_{i}}, N_{x_{j}}\right\rangle \\
& +2 \tau u^{2}\left(\left\langle N_{x_{i}}, N_{x_{j}}\right\rangle\right)_{x_{k}}
\end{aligned}
$$

Then for $0 \leq \tau \leq 1$

$$
\left|g_{i j x_{k}}^{\prime}\right| \leq c_{2}\left(|u|+|\nabla u|+|\nabla u|\left|D^{2} u\right|+|u||\nabla u|+|u|^{2}\right) \leq K_{g}(|u|+|\nabla u|)
$$

which gives us e).

The proofs of $1 \mathrm{~g}$ )-11) are done exactly in the same way with $g=g_{11} g_{22}-$ $g_{12}^{2}$ combining with 1a)-1f).

We have (see lemma 7.1):

$$
b_{i j}(\tau)=\left\langle\psi_{x_{1}}(\tau) \wedge \psi_{x_{2}}(\tau), \psi_{x_{i} x_{j}}(\tau)\right\rangle
$$


Then

$$
\begin{aligned}
\psi_{x_{1}}(\tau) \wedge \psi_{x_{2}}(\tau)= & \left(\psi_{x_{1}} \wedge \psi_{x_{2}}\right)+\tau u\left(\psi_{x_{1}} \wedge N_{x_{2}}+N_{x_{1}} \wedge \psi_{x_{2}}\right) \\
& +\tau u_{x_{1}}\left(N \wedge \psi_{x_{2}}\right)+\tau u_{x_{2}}\left(\psi_{x_{1}} \wedge N\right) \\
& +\tau^{2} u^{2}\left(N_{x_{1}} \wedge N_{x_{2}}\right)+\tau^{2} u u_{x_{2}}\left(N_{x_{1}} \wedge N\right) \\
& +\tau^{2} u u_{x_{1}}\left(N \wedge N_{x_{2}}\right) \\
\psi_{x_{i} x_{j}}(\tau)= & \psi_{x_{i} x_{j}}+\tau u N_{x_{i} x_{j}}+\tau u_{x_{j}} N_{x_{i}}+\tau u_{x_{i}} N_{x_{j}}+\tau u_{x_{i} x_{j}} N
\end{aligned}
$$

Thus

$$
\begin{aligned}
\left|\psi_{x_{i} x_{j}}(\tau)\right| & \leq c_{3} \\
\left|\psi_{x_{i} x_{j}}(\tau)^{\prime}\right| & \leq c_{4}\left(|u|+|\nabla u|+\left|D^{2} u\right|\right) \\
\left|\psi_{x_{i} x_{j}}(\tau)^{\prime \prime}\right| & =0 \\
\left|\psi_{x_{1}}(\tau) \wedge \psi_{x_{2}}(\tau)\right| & \leq c_{5} \\
\left|\left(\psi_{x_{1}}(\tau) \wedge \psi_{x_{2}}(\tau)\right)^{\prime}\right| & \leq c_{6}(|u|+|\nabla u|) \\
\left|\left(\psi_{x_{1}}(\tau) \wedge \psi_{x_{2}}(\tau)\right)^{\prime \prime}\right| & \leq c_{7}\left(|u|^{2}+|u||\nabla u|\right)
\end{aligned}
$$

Thus we get $1 \mathrm{~m})-10$ ) and $1 \mathrm{~s})-1 \mathrm{u}$ ) by derivation of $b_{i j}(\tau)$ like for $g_{i j}(\tau)$.

Moreover

$$
\begin{aligned}
\left|\psi_{x_{i} x_{j} x_{k}}(\tau)\right| & \leq c_{8}\left(1+\left|D^{3} u\right|\right) \\
\left|\psi_{x_{i} x_{j} x_{k}}(\tau)^{\prime}\right| & \leq c_{9}\left(|u|+|\nabla u|+\left|D^{2} u\right|+\left|D^{3} u\right|\right) \\
\left|\psi_{x_{i} x_{j} x_{k}}(\tau)^{\prime \prime}\right| & =0 \\
\left|\left(\psi_{x_{1}}(\tau) \wedge \psi_{x_{2}}(\tau)\right)_{x_{k}}\right| & \leq c_{10} \\
\left|\left(\psi_{x_{1}}(\tau) \wedge \psi_{x_{2}}(\tau)\right)_{x_{k}}^{\prime}\right| & \leq c_{11}\left(|u|+|\nabla u|+\left|D^{2} u\right|\right) \\
\left|\left(\psi_{x_{1}}(\tau) \wedge \psi_{x_{2}}(\tau)\right)_{x_{k}}^{\prime \prime}\right| & \leq c_{12}\left(|u|^{2}+|\nabla u|^{2}+|u|\left|D^{2} u\right|\right)
\end{aligned}
$$

Then the proof of $1 \mathrm{p})-1 \mathrm{r}$ ) and $1 \mathrm{v}$ )-1x) are done exactly in the same way with $b=b_{11} b_{22}-b_{12}^{2}$.

\section{References.}

[1] W.K. Allard, On the first variation of a varifold, Ann. Math. 95 (1972), 417491.

[2] B. Guan and J. Spruck, Boundary Value Problem on $S^{n}$ for surfaces of constant Gauss curvature, Ann. of Math. (2) 138 (1993), No.3, 601-624. 
[3] H. Brezis and J.M. Coron, Multiple solutions of H-systems and Rellich's conjecture, Comm. pure appl. Math. 37 (1984), 149-187.

[4] L.A. Cafarelli, L. Nirenberg, and J. Spruck, The Dirichlet problem for nonlinear second order elliptic equations, I: Monge-Ampère equations, Comm. pure appl. Math. 37 (1984), 369-402.

[5] R. Courant, Dirichlet's principle, conformal mapping, and minimal surfaces, Interscience, 1950.

[6] M. DoCarmo, Differential Geometry of curves and surfaces, Eaglewood Cliffs, New Jersey.

[7] W. Fleming, An example in the problem of least area, Proc. Amer. Math. Society 7 (1956), 1063-1074.

[8] D. Gilbarg and N.S. Trudinger, Elliptic Partial Differential Equations of second order, Berlin Heidelberg New York, Springer 1977.

[9] J. Hass, The geometry of the slice-ribbon problem, Math. Proc. Camb. Phil. Soc. 94 (1983), 101-108.

[10] P. Hall, Two topological examples in minimal surface theory, J. Differential Geom. 19 (1984), 475-481.

[11] D. Hoffman, H. Rosenberg, and J. Spruck, Boundary value problems for surfaces of constant Gauss curvature, Comm. pure and appl. Math. 45 (1992), 1051-1062.

[12] N. Kapouleas, Complete constant mean curvature surfaces in euclidean threespace, Ann. of Math. 131 (1990), 239-330.

[13] B. Lawson, Lectures on minimal submanifolds, vol 1, Publish or Perish, Inc., 1976.

[14] P. Lévy, Le problème de Plateau, Math. Timisoara(Cluj) 23 (1948), 1-45.

[15] H.B. Lawson and M.L. Michelsohn, Approximation by positive mean curvature immersions: frizzing, Invent. Math. 77 (1984), 421-426.

[16] W.H. Meeks and S-T. Yau, The existence of embedded minimal surfaces and the problem of uniqueness, Math. Z. 179 (1982), 151-168.

[17] J.H. Michael and L. Simon, Sobolev and mean value inequalities on generalized submanifolds of $\mathbb{R}^{n}$, Comm. Pure Appl. Math. 26 (1983), 361-397.

[18] C.B. Morrey, Multiple Integrals in calculus of Variations, Berlin Heidelberg New York: Springer 1966. 
[19] H. Rosenberg, Hypersurfaces of constant curvature in space forms, Bull. Sci. Math. 117 (1993). 211-239.

[20] N. Smale, A bridge principle in geometry, Invent. Math. 90 (1987), 505-549.

[21] M. Struwe, Large H-surfaces via the montain pass Lemma, Math. Ann. 270 (1985), 441-459.

[22] M. Traizet, Construction de surfaces minimales en recollant des surfaces de Scherk, Thèse Paris VII(1996).

[23] B. White, The bridge principle for stable minimal surfaces, Cal. Var. Partial Differential Equations 2 (1994), No.4, 405-425.

UNIVERSITY PARIS VII

E-mail address: laurent@math.jussieu.fr 